\title{
Classical vs. non-Archimedean analysis: an approach via algebraic genericity
}

\section{J. Fernández-Sánchez ${ }^{1}$. S. Maghsoudi ${ }^{2}$. D. L. Rodríguez-Vidanes ${ }^{3}$ (D) J. B. Seoane-Sepúlveda ${ }^{4}$}

Received: 27 May 2021 / Accepted: 3 January 2022 / Published online: 29 January 2022

(c) The Author(s) 2022

\begin{abstract}
In this paper, we show new results and improvements of the non-Archimedean counterpart of classical analysis in the theory of lineability. Besides analyzing the algebraic genericity of sets of functions having properties regarding continuity, discontinuity, Lipschitzianity, differentiability and analyticity, we also study the lineability of sets of sequences having properties concerning boundedness and convergence. In particular we show (among several other results) the algebraic genericity of: (i) functions that do not satisfy Liouville's theorem, (ii) sequences that do not satisfy the classical theorem of Cèsaro, or (iii) functionals that do not satisfy the classical Hahn-Banach theorem.
\end{abstract}

Keywords $p$-adic numbers $\cdot p$-adic continuous function $\cdot p$-adic differentiable function . $p$-adic sequences $\cdot$ lineability $\cdot$ algebrability $\cdot$ spaceability $\cdot$ Cesàro summable $\cdot$

non-absolutely convergent series · Liouville's theorem · Lipschitz condition · Hahn-Banach theorem

Mathematics Subject Classification $15 \mathrm{~A} 03 \cdot 46 \mathrm{~B} 87 \cdot 26 \mathrm{E} 30 \cdot 46 \mathrm{~S} 10 \cdot 32 \mathrm{P} 05$

\section{Introduction and preliminaries}

Throughout this paper, we are concerned with the study of rich algebraic structures within families of functions and sequences that are non-linear. This kind of study belongs to the area of lineability theory (intruduced by V. I. Gurariy in the early 2000's [6,31,45], and recently introduced by the AMS under classifications $15 \mathrm{~A} 03$ and 46B87).

D. L. Rodríguez-Vidanes and J. B. Seoane-Sepúlveda were supported by Grant PGC2018-097286-B-I00.D. L. Rodríguez-Vidanes was also supported by the Spanish Ministry of Science, Innovation and Universities and the European Social Fund through a "Contrato Predoctoral para la Formación de Doctores, 2019" (PRE2019-089135). S. Maghsoudi was supported by the Iran National Science Foundation (INSF) Grant no. 99019850.

J. B. Seoane-Sepúlveda jseoane@ucm.es

Extended author information available on the last page of the article 
The one result that, likely, inspired the introduction of this theory was perhaps that of Levine and Milman [39] in 1940, which states that the set of all functions of bounded variation on $[0,1]$ does not contain a closed infinite dimensional subspace in $\mathcal{C}([0,1])$ endowed with the supremum norm.

Later, in 1966, V. I. Gurariy ([32], see also [33]), showed that the set of nowhere differentiable functions on the unit interval of the real line contains an infinite dimensional vector space. These works, among others, motivated the introduction of the notion of lineability in 2005 [6] (notion coined by V. I. Gurariy). One can go further and not just consider linear spaces but, also, larger or more complex structures. For instance, we can name algebrability and strong algebrability defined in $[5,8]$, respectively. We refer the interested reader to [1,2,4-6,10-14,14-17,19-22,24,25,27-29,45] for a current state of the art on this topic.

Before carrying on with the main results of this manuscript, let us gather some necessary definitions and results from lineability theory. We shall use standard set-theoretical notation. As usual, $\mathbb{P}, \mathbb{N}, \mathbb{N}_{0}, \mathbb{Z}, \mathbb{Q}, \mathbb{R}$ and $\mathbb{C}$ denote the sets of all prime numbers, natural, natural numbers including zero, integer, rational, real, and complex numbers, respectively. We identify each cardinal number with the first ordinal of the same cardinality (thus, a cardinal $\kappa$ is equal to the set of all ordinals less than $\kappa$, denoted $\kappa=\{\alpha: \alpha<\kappa\})$. Also, $\aleph_{0}$ and $\mathfrak{c}$ denote the cardinalities of $\mathbb{N}$ and $\mathbb{R}$, respectively. The restriction of a function $f$ to a set $A$ will be denoted by $f \uparrow A$.

We shall frequently use the Fichtenholz-Kantorovich-Hausdorff theorem about families of independent sets in our proofs. This states that for any infinite set $X$ there exists a family $\mathcal{Y} \subseteq \mathcal{P}(X)$ (called a family of independent sets) of cardinality $2^{\text {card }(X)}$ such that for any finite sequences $Y_{1}, \ldots, Y_{n} \in \mathcal{Y}$ and $\varepsilon_{1}, \ldots, \varepsilon_{n} \in\{0,1\}$ we have $Y_{1}^{\varepsilon_{1}} \cap \cdots \cap Y_{n}^{\varepsilon_{n}} \neq \emptyset$, where $Y^{1}=Y$ and $Y^{0}=X \backslash Y$, the complement of the set $Y$ denoted $Y^{c}$. Moreover, all the sets in $\mathcal{Y}$ can be chosen with cardinality $\operatorname{card}(X)$; for more information see, for example, [26,34]. Here $\mathcal{P}(X)$ denotes the power set of $X$. In what follows we fix $\mathcal{P}, \mathcal{N}$ and $\mathcal{B}$ for a family of independent subsets of $\mathbb{P}, \mathbb{N}$ and $[0,1]$, respectively.

Now, we recall some usual terminology from the lineability theory. We say that a subset $A$ of a vector space $V$ over a field $\mathbb{K}$ is:

- $\kappa$-lineable in $V$ (where $\kappa$ is a cardinal number) if there exists a vector space $M$ of dimension $\kappa$ and $M \backslash\{0\} \subseteq A$.

If, in addition, $V$ is a topological vector space over $\mathbb{K}$, then $A$ is said to be:

- $\kappa$-spaceable in $V$ if there exists a closed vector space $M$ of dimension $\kappa$ such that $M \backslash\{0\} \subseteq A$.

Finally, and following [5,8], if $V$ is a topological vector space contained in a (not necessarily unital) algebra and if $\kappa$ and $\beta$ are any (finite or infinite) cardinal numbers, then $A$ is called:

- $\kappa$-algebrable if there exists an algebra $M$ such that $M \backslash\{0\} \subseteq A$ and $M$ is a $\kappa$-dimensional vector space.

- $(\kappa, \beta)$-algebrable if there is an algebra $M$ such that $M \backslash\{0\} \subseteq A, \operatorname{dim}(M)=\kappa$ and there exists a minimal subset $S$ of generators for the algebra $M$ such that $\operatorname{card}(S)=\beta$.

Here, by $S=\left\{s_{\alpha}: \alpha \in I\right\}$ is a minimal system of generators of $M$, we mean that $M$ is the algebra generated by $S$ and for every $\alpha_{0} \in I, s_{\alpha_{0}}$ does not belong to the algebra generated by $S \backslash\left\{s_{\alpha_{0}}\right\}$.

- strongly $\kappa$-algebrable if there exists a $\kappa$-generated free algebra $M$ such that $M \backslash\{0\} \subseteq A$. 
Recall that an algebra $M$ is called a $\kappa$-generated free algebra if there exists a subset $X=\left\{x_{\alpha}: \alpha<\kappa\right\}$ of $M$ such that any function $f$ from $X$ to some algebra $\mathfrak{A}$ can be uniquely extended to a homomorphism from $M$ into $\mathfrak{A}$. Then $X$ is called a set of free generators of the algebra $M$. In a commutative algebra we have a simple criterion; namely, a subset $X=$ $\left\{x_{\alpha}: \alpha<\kappa\right\}$ in a commutative algebra $B$ generates a free subalgebra $M$ if and only if for any polynomial $P$ without free term and any $x_{\alpha_{i}} \in X, 1 \leq i \leq n$, we have $P\left(x_{\alpha_{1}}, \ldots, x_{\alpha_{n}}\right)=0$ if and only if $P=0$. It should be noted that $X=\left\{x_{\alpha}: \alpha<\kappa\right\} \subset B$ is a set of free generators of a free algebra $M \subset B$ if and only if the set of all elements of the form $x_{\alpha_{1}}^{k_{1}} x_{\alpha_{2}}^{k_{2}} \cdots x_{\alpha_{n}}^{k_{n}}$ is linearly independent and all linear combinations of these elements (called algebraic combinations) are in $B \cup\{0\}$. The notion of strong algebrability is essentially stronger than the notion of algebrability; see [8].

Recently, we began to study the lineability of certain subsets of functions and sequences over valued fields different from the classical fields of real and complex numbers; see [23,3638] . In this work we continue this study, investigating lineability and other related notions for the spaces of functions and sequences over the field of $p$-adic numbers in order to establish and compare the analogous of recent results within the context of non-Archimedean analysis. This ought to give a new insight in the theory of lineability in particular and in analysis in general by showing what properties of the scalar field are crucial for classical results.

This paper is arranged as follows. In Sect. 2, we recall some standard concepts and notations concerning non-Archimedean analysis. In Sect. 3, we first show that, if $\mathbb{K} \in$ $\left\{\mathbb{Q}_{p}, \overline{\mathbb{Q}_{p}}, \mathbb{C}_{p}, \Omega_{p}\right\}$, the set of everywhere surjective functions from $\mathbb{K}$ to $\mathbb{K}$ is $2^{\mathfrak{c}}$-lineable, and $\left(2^{\mathfrak{c}}, 2^{\mathfrak{c}}\right)$-algebrable when $\mathbb{K} \neq \mathbb{Q}_{p}$; generalizing [7] to the most important non-Archimedean fields. Some results about Darboux continuity and functions having continuity only at a fixed closed proper set are also given. Then we prove that the set of Lipschitz functions of order $1 / m$ which are not of order $1 /(m-1)$ for any integer $m \geq 2$ is $\mathfrak{c}$-lineable (obtaining as a consequence an improvement of [37] about the set of continuous nowhere differentiable functions). We also show that the set of discontinuous functions with finite range that have antiderivative and the set of separately continuous functions from $\mathbb{Q}_{p}^{n}$ to $\mathbb{Q}_{p}$ with $p>2$ and $n \geq 2$ that are everywhere continuous except at a point are c-lineable. In Sect. 4, we begin by showing that the set of functions on $\mathbb{Q}_{p}$ that do not satisfy Liouville's theorem is $\mathfrak{c}$-lineable. Then we prove a similar result for the set of sequences of functions that do not satisfy a well known classical theorem on the interchange of limit and derivative, and another result involving continuity and differentiablity. We also study the lineability of the set of non-locally constant functions that have derivative 0 with additional Lipschitz conditions. Finally, in Sect. 5, we provide some results on the spaces of $p$-adic sequences. More specifically, we show that the set of bounded sequences not converging to zero is $\mathfrak{c}$-spaceable and, within the same set, the family of sequences that also have only finitely many zero coordinates is strongly $\mathfrak{c}$-algebrable. We also prove the strong $\mathfrak{c}$-algebrability of the set of non-absolutely convergent series that are convergent; a result without any counterpart in the real case. In the same line of study, we also prove that the set of convergent sequences that are not Cesàro summable is strongly $\mathfrak{c}$-algebrable. Finally, we establish that the family of functionals on $c_{0}$, considered over any non-spherically complete non-Archimedean field with non-trivial valuation, that do not have any continuous extension on $\ell^{\infty}$ is $\mathfrak{c}$-lineable. That means that the set of functionals that do not satisfy the classical Hahn-Banach theorem in the non-Archimedean setting is algebraically generic. Comparing with the classical cases, these results require an entirely new approach for their proofs. 


\section{A brief background $p$-adic analysis}

We refer the interested reader for a more profound treatment of these topics to $[4,30,35,43$, $44,46]$. Now we turn to the main object of the paper; $p$-adic analysis. A non-Archimedean field is a field $\mathbb{K}$ equipped with a function (valuation)

$$
|\cdot|: \mathbb{K} \rightarrow[0, \infty)
$$

such that:

- $|x|=0$ if and only if $x=0$,

- $|x y|=|x||y|$, and

- $|x+y| \leq \max \{|x|,|y|\}$ (the strong triangle inequality),

for all $x, y \in \mathbb{K}$. Clearly, $|1|=|-1|=1$ and the valuation of summing $n$-times 1 is less or equal than 1 for all $n \in \mathbb{N}$. An immediate consequence of the so-called strong triangle inequality is that $|x| \neq|y|$ implies $|x+y|=\max \{|x|,|y|\}$. A trivial example of a nonArchimedean valuation is the function $|\cdot|$ taking everything except 0 into 1 and $|0|=0$. This valuation is referred to as the "trivial" one. By Big Ostrowski's theorem (see [46, theorem 1.2]) any complete valued field $\mathbb{K}$ that is not topologically isomorphic to $\mathbb{R}$ or $\mathbb{C}$ is non-Archimedean.

Let us denote by $p$ an arbitrary prime number throughout this work. For any non-zero integer $n \neq 0$, let $\operatorname{ord}_{p}(n)$ be the highest power of $p$ which divides $n$. Then we define $|n|_{p}=p^{-\operatorname{ord}_{p}(n)},|0|_{p}=0$ and $\left|\frac{n}{m}\right|_{p}=p^{-\operatorname{ord}_{p}(n)+\operatorname{ord}_{p}(m)}$. The completion of the field of rationals, $\mathbb{Q}$, with respect to the $p$-adic metric $d(x, y)=|x-y|_{p}$ is called the field of $p$-adic numbers $\mathbb{Q}_{p}$. The metric $d$ satisfies the strong triangle inequality $|x \pm y|_{p} \leq \max \left\{|x|_{p},|y|_{p}\right\}$. Ostrowski's theorem states that every non-trivial absolute value on $\mathbb{Q}$ is equivalent (i.e., defines the same topology) to an absolute value $|\cdot|_{p}$, for some prime number $p$, or the usual absolute value (see [30]).

Let $a \in \mathbb{Q}_{p}$ and $r$ be a positive number. The set $B(a, r)=\left\{x \in \mathbb{Q}_{p}:|x-a|_{p}<r\right\}$ is called the open ball of radius $r$ with center $a, \bar{B}(a, r)=\left\{x \in \mathbb{Q}_{p}:|x-a|_{p} \leq r\right\}$ the closed ball of radius $r$ with center $a$, and $S(a, r)=\left\{x \in \mathbb{Q}_{p}:|x-a|_{p}=r\right\}$ the sphere of radius $r$ and center $a$. The ring of integers in $\mathbb{Q}_{p}$ is denoted by $\mathbb{Z}_{p}$, i.e., $\mathbb{Z}_{p}=\left\{x \in \mathbb{Q}_{p}:|x|_{p} \leq 1\right\}$. Note that every $x \in \mathbb{Z}_{p}$ can be expanded in canonical form as $x=a_{0}+a_{1} p+\cdots+a_{k} p^{k}+\cdots$, $a_{k} \in\{0,1, \ldots, p-1\}, k \geq 0$. We know that $\mathbb{Z}_{p}$ is a compact set and $\mathbb{N}$ is dense in $\mathbb{Z}_{p}$ [30]. Note that $\mathbb{Q}_{p}$ is an infinite dimensional vector space over $\mathbb{Q}$. In view of the fact that $\operatorname{card}\left(\mathbb{Z}_{p} \backslash\{0\}\right)=\mathfrak{c}$ and by applying the canonical representation of the $p$-adic rationals, we can take a Hamel basis of $\mathbb{Q}_{p}$ over $\mathbb{Q}$ contained in $\mathbb{Z}_{p} \backslash\{0\}$.

As usual, we also denote by $\overline{\mathbb{Q} p}$ the algebraic closure of $\mathbb{Q}_{p}$ and by $\mathbb{C}_{p}$ the completion of $\overline{\mathbb{Q}_{p}}$ with respect to the extended $p$-adic valuation. Finally, by $\Omega_{p}$ we denote the spherically complete extension of $\mathbb{C}_{p}$; see [43, III.2]. A metric space is called spherically complete if each nested sequence of balls has a non-empty intersection.

Let us remark that the derivative of $p$-adic functions and analyticity of functions is defined as in the case of classical real functions, for more details, see [46]. Other functions that are relevant for our purposes are the Lipschitz functions. For any $\alpha>0$, the space of Lipschitz functions from $\mathbb{K}_{1}$ to $\mathbb{K}_{2}$ of order $\alpha$ is defined as 
$\operatorname{Lip}_{\alpha}\left(\mathbb{K}_{1} \rightarrow \mathbb{K}_{2}\right):=\left\{f: \mathbb{K}_{1} \rightarrow \mathbb{K}_{2}: \exists M>0\left(|f(x)-f(y)|_{p} \leq M|x-y|_{p}^{\alpha}, \forall x, y \in \mathbb{K}_{1}\right)\right\}$, where $\mathbb{K}_{1} \in\left\{\mathbb{Z}_{p}, \mathbb{Q}_{p}\right\}$ and $\mathbb{K}_{2} \in\left\{\mathbb{Q}_{p}, \overline{\mathbb{Q}_{p}}, \mathbb{C}_{p}, \Omega_{p}\right\}$.

The binomial coefficient functions $\left(\begin{array}{l}x \\ n\end{array}\right)$ are defined for $x \in \mathbb{Z}_{p}$ and $n \in \mathbb{N}_{0}$ by

$$
\left(\begin{array}{l}
x \\
n
\end{array}\right)= \begin{cases}\frac{x(x-1) \cdots(x-n+1)}{n !} & \text { if } n>0 \\
1 & \text { if } n=0\end{cases}
$$

Finally, we define $\mathbb{K}$-normed spaces. Let $V$ be a linear space over a field $\mathbb{K}$ with a nonArchimedean non-trivial valuation $|\cdot|$. A function $\|\cdot\|: V \rightarrow[0, \infty)$ is said to be a non-Archimedean norm on $V$ if the following conditions hold:

(i) $\|x\|=0$ if and only if $x=0$,

(ii) $\|\lambda x\|=|\lambda|\|x\|$, for all $\lambda \in \mathbb{K}$ and $x \in V$,

(iii) $\|x+y\| \leq \max \{\|x\|,\|y\|\}$, for all $x, y \in V$.

Then $(V,\|\cdot\|)$ is called a non-Archimedean normed space or a normed space over $\mathbb{K}$. When $V$ is complete with respect to the norm $\|\cdot\|$, it is called Banach space over $\mathbb{K}$.

The Banach space of all sequences $\left(x_{n}\right)_{n \geq 0}$ with coordinates in $\mathbb{K}$ such that $\left\|\left(x_{n}\right)_{n \geq 0}\right\|_{\infty}:=$ $\sup _{n \in \mathbb{N}_{0}}\left|x_{n}\right| \leq M$, for some $M \geq 0$, is denoted by $\ell^{\infty}$. The subspace of $\ell^{\infty}$ consisting of all sequences converging to zero is denoted by $c_{0}$. Unlike the Archimedean world, the dual space of $c_{0}$ is isometrically isomorphic to $\ell^{\infty}$ (see [42, theorem 2.5.11]).

Throughout this article we shall consider all vector spaces and algebras taken over the field $\mathbb{K}=\mathbb{Q}_{p}$ (unless stated otherwise).

\section{Algebraic genericity of sets of $p$-adic discontinuous, continuous, and Lipschitz functions}

We begin this section with a result about everywhere surjective functions. For a topological space $X$ and a non-empty set $Y$, we say that $f: X \rightarrow Y$ is everywhere surjective (ES) provided $f[U]=Y$ for every non-empty open subset $U$ of $X$. Apparently, the first example of these surprising functions on the real line is due to H. Lebesgue. Algebrability of these functions and other variants have been studied; see for example [7,9]. We give an optimal result in the sense of cardinality for the considered valued fields. To do this let us consider the following partition of $\mathbb{Q}_{p}$ into $\mathfrak{c}$-many sets of cardinality $\mathfrak{c}$. For every $\alpha \in[0,1]$, define

$$
A_{\alpha}=\left\{x=\sum_{i=-m}^{\infty} x_{i} p^{i} \in \mathbb{Q}_{p}: \limsup _{n \rightarrow \infty} \frac{\operatorname{card}\left(\left\{x_{2 k}=0: 1 \leq k \leq n\right\}\right)}{n}=\alpha\right\} .
$$

Now, partition $A_{\alpha}$ into $\mathfrak{c}$-many sets of cardinality $\mathfrak{c}$ as follows: for every $\beta \in[0,1]$, define

$$
A_{\alpha, \beta}=\left\{x \in A_{\alpha}: \limsup _{n \rightarrow \infty} \frac{\operatorname{card}\left(\left\{x_{2 k-1}=0: 1 \leq k \leq n\right\}\right)}{n}=\beta\right\} .
$$

Theorem 3.1 If $\mathbb{K} \in\left\{\mathbb{Q}_{p}, \overline{\mathbb{Q}_{p}}, \mathbb{C}_{p}, \Omega_{p}\right\}$, then the family of ES functions $\mathbb{Q}_{p} \rightarrow \mathbb{K}$ is

(i) $2^{\mathfrak{c}}$-lineable (as a $\mathbb{K}$-vector space),

(ii) $\left(2^{\mathfrak{c}}, 2^{\mathfrak{c}}\right)$-algebrable if $\mathbb{K} \neq \mathbb{Q}_{p}$ (as a $\mathbb{K}$-vector space). 
Proof Fix $\mathbb{K} \in\left\{\mathbb{Q}_{p}, \overline{\mathbb{Q}_{p}}, \mathbb{C}_{p}, \Omega_{p}\right\}$. Since card $(\mathbb{K})=\mathfrak{c}$, let $\gamma:[0,1] \rightarrow \mathbb{K}$ be a bijection. Also let us define for every $\alpha \in[0,1]$ the function $r_{\alpha}: \mathbb{Q}_{p} \rightarrow \mathbb{K}$ as follows: for every $x \in \mathbb{Q}_{p}$,

$$
r_{\alpha}(x)= \begin{cases}\gamma(\beta) & \text { if } x \in A_{\alpha, \beta}, \\ 0 & \text { otherwise. }\end{cases}
$$

The functions $r_{\alpha}$ are ES. Indeed, fix $a \in \mathbb{Q}_{p}$ and $\varepsilon \in\left\{p^{n}: n \in \mathbb{Z}\right\}$. It is enough to prove that $r_{\alpha}[B(a, \varepsilon)]=\mathbb{K}$. Take $y \in \mathbb{K}$ arbitrary, then there exists a unique $\beta \in[0,1]$ such that $\gamma(\beta)=y$. Now choose $x \in B(a, \varepsilon) \backslash\{a\}$, then $0<|x-a|_{p}=p^{t}<p^{n}$ for some $t \in \mathbb{Z} \backslash\{0\}$.

Case (1): If $t>0$, then change the coefficients of $p^{k}$ where $k \geq 0$ in the canonical representation of $x$ so that $x \in A_{\alpha, \beta}$.

Case (2): If $t \leq 0$, then change the coefficients of $p^{k}$ where $k>t$ in the canonical representation of $x$ so that $x \in A_{\alpha, \beta}$.

Notice that in both cases we have $x \in B(a, \varepsilon) \cap A_{\alpha, \beta}$. Hence, $r_{\alpha}(x)=y$.

For every $B \in \mathcal{B}$, we define $r_{B}=\sum_{\alpha \in B} r_{\alpha}$. We will prove first that the functions $r_{B}$ are well defined for any $B \in \mathcal{B}$. For every $x \in \mathbb{Q}_{p}$, there exist unique $\alpha, \beta \in[0,1]$ such that $x \in A_{\alpha, \beta}$. If $\alpha \in B$, then $r_{B}(x)=\gamma(\beta)$ since $\left\{A_{\alpha}: \alpha \in \mathbb{Q}_{p}\right\}$ forms a partition of $\mathbb{Q}_{p}$ and $\left\{A_{\alpha, \beta}: \beta \in \mathbb{Q}_{p}\right\}$ forms a partition of $A_{\alpha}$. If not, then $r_{B}(x)=0$. Clearly, the function $r_{B}$ is ES for every $B \in \mathcal{B}$ since for any $\alpha \in B$ and any non-empty open subset $U$ of $\mathbb{Q}_{p}$ we have $\mathbb{K} \supseteq r_{B}[U] \supseteq r_{B}\left[U \cap A_{\alpha}\right]=r_{\alpha}\left[U \cap A_{\alpha}\right]=\mathbb{K}$.

Let $r=\sum_{i=1}^{n} a_{i} r_{B_{i}}$ with $n \in \mathbb{N}, a_{i} \in \mathbb{K} \backslash\{0\}$ for every $1 \leq i \leq n$ and $B_{1}, \ldots, B_{n} \in \mathcal{B}$ distinct. Choose $\alpha \in B_{1} \cap B_{2}^{c} \cap \cdots \cap B_{n}^{c}$. Thus, for every $x \in A_{\alpha}$, we have $r(x)=a_{1} r_{B_{1}}(x)$. Hence, the functions $\left\{r_{B}: B \in \mathcal{B}\right\}$ are linearly independent over $\mathbb{K}$. Moreover, since $\mathbb{K} \supseteq$ $r[U] \supseteq r\left[U \cap A_{\alpha}\right]=a_{1} r_{B_{1}}\left[U \cap A_{\alpha}\right]=a_{1} \mathbb{K}=\mathbb{K}$ for any non-empty open subset $U$ of $\mathbb{Q}_{p}$, we have that $r$ is ES.

Assume now that $\mathbb{K} \neq \mathbb{Q}_{p}$. Let us prove first that $r_{B_{1}}$ does not belong to the algebra generated by $\left\{r_{B}: B \in \mathcal{B}\right\} \backslash\left\{r_{B_{1}}\right\}$. Assume otherwise, that is, there exist $B_{2}, \ldots, B_{n} \in \mathcal{B} \backslash\left\{B_{1}\right\}$ distinct and a polynomial $P$ in $n-1$ variables with coefficients in $\mathbb{K} \backslash\{0\}$ and without free term such that $r_{B_{1}}=P\left(r_{B_{2}}, \ldots, r_{B_{n}}\right)$. Take $\alpha \in B_{1} \cap B_{2}^{c} \cap \cdots \cap B_{n}^{c}$, then for every $x \in A_{\alpha}$ we have

$$
r_{\alpha}(x)=r_{B_{1}}(x)=P\left(r_{B_{2}}(x), \ldots, r_{B_{n}}(x)\right)=P(0, \ldots, 0) .
$$

However, since $r_{\alpha}\left[A_{\alpha}\right]=\mathbb{K}$, we have reached a contradiction.

Now let $B_{1}, \ldots, B_{n} \in \mathcal{B}$ be distinct. Assume that $P$ is a polynomial in $n$ variables with coefficients in $\mathbb{K} \backslash\{0\}$ and without free term such that $P\left(r_{B_{1}}\left(x_{0}\right), \ldots, r_{B_{n}}\left(x_{0}\right)\right) \neq 0$ for some $x_{0} \in \mathbb{Q}_{p} \backslash\{0\}$. Then there exists $\alpha \in B_{1} \cup \cdots \cup B_{n}$ such that $x_{0} \in A_{\alpha}$, since otherwise we would have $P\left(r_{B_{1}}\left(x_{0}\right), \ldots, r_{B_{n}}\left(x_{0}\right)\right)=P(0, \ldots, 0)=0$ contradicting the construction of $P$. Thus, for any $x \in A_{\alpha}$, we see that $P\left(r_{B_{1}}(x), \ldots, r_{B_{n}}(x)\right)$ is of the form $P_{1}\left(r_{\alpha}(x)\right)$, where $P_{1}$ is a polynomial in 1 variable with coefficients in $\mathbb{K} \backslash\{0\}$ and without free term. Therefore, as $\mathbb{K} \backslash\{0\}$ is algebraically closed, for every non-empty open set $U$ of $\mathbb{Q}_{p}$ we have

$$
\mathbb{K} \supseteq P\left(r_{B_{1}}, \ldots, r_{B_{n}}\right)[U] \supseteq P\left(r_{B_{1}}, \ldots, r_{B_{n}}\right)\left[U \cap A_{\alpha}\right]=P_{1}\left(r_{\alpha}\right)\left[U \cap A_{\alpha}\right]=\mathbb{K} .
$$

This proves (ii).

A consequence of Theorem 3.1 is the following.

Corollary 3.2 If $\mathbb{K} \in\left\{\overline{\mathbb{Q}_{p}}, \mathbb{C}_{p}, \Omega_{p}\right\}$, then the family of ES functions $\mathbb{K} \rightarrow \mathbb{K}$ is $\left(2^{\mathfrak{c}}, 2^{\mathfrak{c}}\right)$ algebrable. 
Proof Fix $\mathbb{K} \in\left\{\overline{\mathbb{Q}_{p}}, \mathbb{C}_{p}, \Omega_{p}\right\}$ and let $r_{B}: \mathbb{Q}_{p} \rightarrow \mathbb{K}$ be the functions defined in the proof of Theorem 3.1. Take $\mathcal{H}$ be a Hamel basis of $\mathbb{K}$ over $\mathbb{Q}_{p}$ containing 1 . Then, for every $x \in \mathbb{K}$, we have that $x$ can be decomposed as $x=\beta \cdot 1+y=\beta+y$, where $\beta \in \mathbb{Q}_{p}$ and $y \in \operatorname{span}\langle\mathcal{H} \backslash\{1\}\rangle$. For every $B \in \mathcal{B}$, define $\rho_{B}(x)=r_{B}(\beta)$ for every $x=\beta+y \in \mathbb{K}$. Clearly the family of functions $\left\{\rho_{B}: B \in \mathcal{B}\right\}$ is linearly independent. Now take a non-empty open subset $U$ of $\mathbb{K}$ and fix $x=\beta+y \in U$, then there exists $\varepsilon>0$ such that $B(x, \varepsilon) \subseteq U$. Therefore for every $z \in B(\beta, \varepsilon) \cap \mathbb{Q}_{p}$ (where $B(\beta, \varepsilon) \cap \mathbb{Q}_{p}$ is a non-empty open subset of $\left.\mathbb{Q}_{p}\right)$ we have $z+y \in B(x, \varepsilon)$ since $|z+y-x|_{p}=|z+y-\beta-y|_{p}=|z-\beta|_{p}<\varepsilon$. Hence,

$$
\begin{aligned}
\mathbb{K} & \supseteq \rho_{B}[U] \supseteq \rho_{B}[B(x, \varepsilon)] \supseteq \rho_{B}\left[\left\{z+y: z \in B(\beta, \varepsilon) \cap \mathbb{Q}_{p}\right\}\right] \\
& =r_{B}\left[\left\{z: z \in B(\beta, \varepsilon) \cap \mathbb{Q}_{p}\right\}\right]=r_{B}\left[B(\beta, \varepsilon) \cap \mathbb{Q}_{p}\right]=\mathbb{K} .
\end{aligned}
$$

Assume now that $\rho_{B_{1}}$ belongs to the algebra generated by $\left\{\rho_{B}: B \in \mathcal{B}\right\} \backslash\left\{\rho_{B_{1}}\right\}$, then there exist $B_{2}, \ldots, B_{n} \in \mathcal{B} \backslash\left\{B_{1}\right\}$ distinct and a polynomial $P$ in $n-1$ variables with coefficients in $\mathbb{K} \backslash\{0\}$ and without free term such that $\rho_{B_{1}}=P\left(\rho_{B_{2}}, \ldots, \rho_{B_{n}}\right)$. Take $\alpha \in B_{1} \cap B_{2}^{c} \cap \cdots \cap B_{n}^{c}$; and fix an arbitrary ball $B(x, \varepsilon) \in \mathbb{K}$, where $x=\beta+y$. For every $z \in B(\beta, \varepsilon) \cap \mathbb{Q}_{p} \cap A_{\alpha}$, we have

$$
\begin{aligned}
r_{\alpha}(z) & =r_{B_{1}}(z)=\rho_{B_{1}}(z+y)=P\left(\rho_{B_{2}}(z+y), \ldots, \rho_{B_{n}}(z+y)\right) \\
& =P\left(r_{B_{2}}(z), \ldots, r_{B_{n}}(z)\right)=P(0, \ldots, 0)=0 .
\end{aligned}
$$

But, since $r_{\alpha}\left[B(\beta, \varepsilon) \cap \mathbb{Q}_{p} \cap A_{\alpha}\right]=\mathbb{K}$, we have a contradiction.

Finally, take $B_{1}, \ldots, B_{n} \in \mathcal{B}$ distinct and $P$ a polynomial in $n$ variables with coefficients in $\mathbb{K} \backslash\{0\}$ and without free term such that $P\left(\rho_{B_{1}}\left(x_{0}\right), \ldots, \rho_{B_{n}}\left(x_{0}\right)\right) \neq 0$ for some $x_{0} \in \mathbb{K} \backslash\{0\}$. Then there exists $\alpha \in B_{1} \cup \cdots \cup B_{n}$ such that $x_{0}=\beta_{0}+y_{0}$ with $\beta_{0} \in A_{\alpha}$ and $y_{0} \in \operatorname{span}\langle\mathcal{H} \backslash\{1\}\rangle$, since otherwise we would have $P\left(\rho_{B_{1}}\left(x_{0}\right), \ldots, \rho_{B_{n}}\left(x_{0}\right)\right)=$ $P(0, \ldots, 0)=0$. Thus, for any $x=\beta+y \in \mathbb{K}$ with $\beta \in A_{\alpha}$ and $y \in \operatorname{span}\langle\mathcal{H} \backslash\{1\}\rangle$, we see that $P\left(\rho_{B_{1}}(x), \ldots, \rho_{B_{n}}(x)\right)$ is of the form $P_{1}\left(r_{\alpha}(x)\right)$, where $P_{1}$ is a polynomial in 1 variable with coefficients in $\mathbb{K} \backslash\{0\}$ and without free term. Let $U$ be a non-empty subset of $\mathbb{K}$ and take an arbitrary open ball $B(x, \varepsilon) \subset U$, where $x=\beta+y$. Therefore, as $\mathbb{K} \backslash\{0\}$ is algebraically closed, we have

$$
\begin{aligned}
\mathbb{K} & \supseteq P\left(\rho_{B_{1}}, \ldots, \rho_{B_{n}}\right)[U] \supseteq P\left(\rho_{B_{1}}, \ldots, \rho_{B_{n}}\right)[B(x, \varepsilon)] \\
& \supseteq P\left(\rho_{B_{1}}, \ldots, \rho_{B_{n}}\right)\left[\left\{z+y: z \in B(\beta, \varepsilon) \cap \mathbb{Q}_{p} \cap A_{\alpha}\right\}\right] \\
& =P_{1}\left(r_{\alpha}\right)\left[B(\beta, \varepsilon) \cap \mathbb{Q}_{p} \cap A_{\alpha}\right]=\mathbb{K} .
\end{aligned}
$$

Let us recall that a subset $C$ of a non-Archimedean field $\mathbb{K}$ is called convex if $\lambda x+\mu y+v z \in$ $C$ for every $x, y, z \in C$, and $\lambda, \mu, v \in \mathbb{K}$ with $|\lambda|,|\mu|,|\nu| \leq 1$ and $\lambda+\mu+v=1$ (see [42, theorem 3.1.15]). It can be seen that the only convex subsets of $\mathbb{K}$ are $\emptyset, \mathbb{K}$, the singleton sets and balls (see [42, p. 89]). A function $f: C \rightarrow \mathbb{K}$, where $C$ is a convex set, is called (convex) Darboux continuous if for each convex set $A \subseteq \mathbb{K}$, the image $f[A]$ is convex in $\mathbb{K}$.

Remark 3.3 Notice that the definition of convex set over a non-Archimedean field $\mathbb{K}$ is not the classical definition of a convex set in the Archimedean fields $\mathbb{R}$ or $\mathbb{C}$. Recall that a subset $C$ of $\mathbb{R}$ (resp. $\mathbb{C}$ ) is convex provided that $\lambda x+\mu y \in C$ for every $x, y \in C$, and $\lambda, \mu \in \mathbb{R}$ (resp. $\mathbb{C}$ ) with $|\lambda|,|\mu| \leq 1$ and $\lambda+\mu=1$. The reader may think that such definition can be adapted to any non-Archimedean field but this is not the case, for instance, on $\mathbb{Q}_{2}$, since the residue class field of $\mathbb{Q}_{2}$ is the finite field of 2 elements $\mathbf{F}_{2}$ (see [42, theorem 3.1.17] and $[43$, p. 145]). 
Take $\mathbb{K} \in\left\{\mathbb{Q}_{p}, \overline{\mathbb{Q}}_{p}, \mathbb{C}_{p}, \Omega_{p}\right\}$. If $f: \mathbb{Q}_{p} \rightarrow \mathbb{K}$ (resp. $f: \mathbb{K} \rightarrow \mathbb{K}$ ) is an ES function and $C$ is a convex subset of $\mathbb{Q}_{p}($ resp. $\mathbb{K})$, then $f[C]$ is the empty set, a singleton set, or $\mathbb{K}$, i.e., a convex subset of $\mathbb{K}$. Hence, as an immediate consequence of Theorem 3.1 and Corollary 3.2, we have the following corollary, which generalizes [38, theorem 2.3].

Corollary 3.4 (1) If $\mathbb{K} \in\left\{\mathbb{Q}_{p}, \overline{\mathbb{Q}_{p}}, \mathbb{C}_{p}, \Omega_{p}\right\}$, then the family of functions $\mathbb{Q}_{p} \rightarrow \mathbb{K}$ that are Darboux continuous but not continuous is

(i) $2^{\mathfrak{c}}$-lineable (as a $\mathbb{K}$-vector space),

(ii) $\left(2^{\mathfrak{c}}, 2^{\mathfrak{c}}\right)$-algebrable if $\mathbb{K} \neq \mathbb{Q}_{p}$ (as a $\mathbb{K}$-vector space).

(2) If $\mathbb{K} \in\left\{\overline{\mathbb{Q}}_{p}, \mathbb{C}_{p}, \Omega_{p}\right\}$, then the family of functions $\mathbb{K} \rightarrow \mathbb{K}$ that are Darboux continuous but not continuous is $\left(2^{\mathfrak{c}}, 2^{\mathfrak{c}}\right)$-algebrable.

The next result generalizes [38, theorem 2.5].

Proposition 3.5 The family of continuous functions $\mathbb{Q}_{p} \rightarrow \mathbb{Q}_{p}$ that are not Darboux continuous is strongly $\mathfrak{c}$-algebrable.

Proof For every $n \in \mathbb{N}_{0}$, we denote by $S_{n}$ the following set:

$$
S_{n}= \begin{cases}\left\{x \in \mathbb{Q}_{p}:|x|=p^{n}\right\} & \text { if } n \geq 1 \\ \left\{x \in \mathbb{Q}_{p}:|x| \leq 1\right\} & \text { otherwise. }\end{cases}
$$

Notice that $\mathbb{Q}_{p}=\bigsqcup_{n \in \mathbb{N}_{0}} S_{n}$. For every $\alpha \in(0,1)$, we define $h_{\alpha}: \mathbb{Q}_{p} \rightarrow \mathbb{Q}_{p}$ as $h_{\alpha}(x)=$ $p^{-\left\lfloor n^{\alpha}\right\rfloor}$ if $x \in S_{n}$ for every $n \in \mathbb{N}_{0}$. Since the spheres and closed balls of the space $\mathbb{Q}_{p}$ are open (see [42, proposition 2.2]), we have that the sets $S_{n}$ are open and, therefore, $h_{\alpha}$ is locally constant for every $\alpha \in(0,1)$. Hence, $h_{\alpha}$ is continuous for every $\alpha \in(0,1)$ and, thus, every algebraic combination of the functions $\left\{h_{\alpha}: \alpha \in(0,1)\right\}$ is also continuous.

Take, without loss of generality, $0<\alpha_{1}<\cdots<\alpha_{k}<1$. Let $P$ be a polynomial in $k$ variables with coefficients in $\mathbb{Q}_{p} \backslash\{0\}$ and without free term. If $P_{i}$ are the monomials that form $P$ and $x \in S_{n}$, then

$$
P\left(h_{\alpha_{1}}, \ldots, h_{\alpha_{k}}\right)(x)=\sum_{i=1}^{t} P_{i}\left(p^{-\left\lfloor n^{\alpha_{1}}\right\rfloor}, \ldots, p^{-\left\lfloor n^{\alpha_{k}}\right\rfloor}\right) .
$$

In particular,

$$
P\left(h_{\alpha_{1}}, \ldots, h_{\alpha_{k}}\right)(x)=\sum_{i=1}^{t} \gamma_{i} p^{-i_{k}\left\lfloor n^{\alpha_{k}}\right\rfloor-\cdots-i_{1}\left\lfloor n^{\alpha_{1}}\right\rfloor},
$$

where $\gamma_{i} \in \mathbb{Q}_{p} \backslash\{0\}$ are the coefficients of $P$ and $i_{k}, \ldots, i_{1}$ are non-negative integers. Assume, without loss of generality, that the $k$-tuples $\left(i_{k}, \ldots, i_{1}\right)$ from (3.1) appear ordered lexicographically. Notice that all the $k$-tuples are distinct since otherwise we could add the monomials in the polynomial $P$ that have these $k$-tuples as exponents. Clearly, we have that $\left(t_{k}, \ldots, t_{1}\right)=\max \left\{\left(i_{k}, \ldots, i_{1}\right): 1 \leq i \leq t\right\}$ and also $\left(i_{k}, \ldots, i_{1}\right)<\left(t_{k}, \ldots, t_{1}\right)$ for any other $k$-tuple $\left(i_{k}, \ldots, i_{1}\right)$ in (3.1) (the latter makes sense if there are 2 or more $k$-tuples, if not then $\left(t_{k} \ldots, t_{1}\right)$ is the only $k$-tuple). Hence, there exists $n_{0} \in \mathbb{N}$ such that for any $n \geq n_{0}$ we have

$$
\left|P\left(h_{\alpha_{1}}, \ldots, h_{\alpha_{k}}\right)(x)\right|_{p}=\left|\gamma_{t}\right|_{p} \cdot p^{t_{k}\left\lfloor n^{\alpha_{k}}\right\rfloor+\cdots+t_{1}\left\lfloor n^{\alpha_{1}}\right\rfloor} .
$$

Thus, $P\left(h_{\alpha_{1}}, \ldots, h_{\alpha_{k}}\right)$ is not identically zero. Moreover, $P\left(h_{\alpha_{1}}, \ldots, h_{\alpha_{k}}\right)\left[\mathbb{Q}_{p}\right]$ is an infinite set. Since the functions $h_{\alpha}$ are constant in $S_{n}$ and the sets $S_{n}$ form a partition of $\mathbb{Q}_{p}$, we have 
that the cardinality of the set $P\left(h_{\alpha_{1}}, \ldots, h_{\alpha_{k}}\right)\left[\mathbb{Q}_{p}\right]$ is $\aleph_{0}$. Hence, as the convex sets have cardinality 0,1 or $\mathfrak{c}$ (see [42, lemma 3.2.1]), we have that $P\left(h_{\alpha_{1}}, \ldots, h_{\alpha_{k}}\right)\left[\mathbb{Q}_{p}\right]$ is not convex, i.e., $P\left(h_{\alpha_{1}}, \ldots, h_{\alpha_{k}}\right)$ is not Darboux continuous.

In [38, proposition 2.1], Khodabendehlou and the second and fourth authors prove that given a closed proper subset $F$ of $\mathbb{Q}_{p}$, the family of functions $\mathbb{Q}_{p} \rightarrow \mathbb{Q}_{p}$ that are continuous only at the points belonging to $F$ is $2^{\mathfrak{c}}$-lineable. Here we give a strong version of the result.

Theorem 3.6 If $\mathbb{K} \in\left\{\overline{\mathbb{Q}}_{p}, \mathbb{C}_{p}, \Omega_{p}\right\}$ and $F$ is a closed proper subset of $\mathbb{K}$, then the family of functions $\mathbb{K} \rightarrow \mathbb{K}$ that are continuous only at the points belonging to $F$ is $\left(2^{\mathfrak{c}}, 2^{\mathfrak{c}}\right)$-algebrable.

Proof Fix $\mathbb{K} \in\left\{\overline{\mathbb{Q}_{p}}, \mathbb{C}_{p}, \Omega_{p}\right\}$. Since card $\left(\mathbb{Z}_{p}\right)=\mathfrak{c}$, let $\xi:[0,1] \rightarrow \mathbb{Z}_{p}$ be a bijection. Also let us define for every $\alpha \in[0,1]$, the function $t_{\alpha}: \mathbb{Q}_{p} \rightarrow \mathbb{Z}_{p}$ as follows: for every $x \in \mathbb{Q}_{p}$,

$$
t_{\alpha}(x)= \begin{cases}\xi(\beta) & \text { if } x \in A_{\alpha, \beta}, \\ 0 & \text { otherwise. }\end{cases}
$$

Following the proof of Theorem 3.1 define the functions $\varphi_{B}=\sum_{\alpha \in B} t_{\alpha}$ for every $B \in \mathcal{B}$. As in the proof of Theorem 3.1, notice that the functions $\varphi_{B}$ are well defined functions from $\mathbb{Q}_{p}$ to $\mathbb{Z}_{p}$ and also $t_{\alpha}\left[U \cap A_{\alpha}\right]=\mathbb{Z}_{p}$ for every non-empty open subset $U$ of $\mathbb{Q}_{p}$.

Take $\mathcal{H}$ a Hamel basis of $\mathbb{K}$ over $\mathbb{Q}_{p}$ containing 1 . Then, for every $x \in \mathbb{K}$, we have that $x$ can be decomposed as $x=\beta \cdot 1+y=\beta+y$, where $\beta \in \mathbb{Q}_{p}$ and $y \in \operatorname{span}\langle\mathcal{H} \backslash\{1\}\rangle$. For every $B \in \mathcal{B}$, define $\phi_{B}(x)=\varphi_{B}(\beta)$ for every $x=\beta+y \in \mathbb{K}$. Similar to the proof of Corollary 3.2, the functions $\phi_{B}$ are ES from $\mathbb{K}$ to $\mathbb{Z}_{p}$.

Consider now the distance function $d^{\prime}(x, F)=\min \left\{|x-c|_{p}: c \in F\right\}$, where $x \in \mathbb{K}$, and take the sets

$$
F_{m}= \begin{cases}\left\{x \in \mathbb{K}: p^{-(m+1)}<d^{\prime}(x, F) \leq p^{-m}\right\} & \text { if } m \in \mathbb{N}, \\ \left\{x \in \mathbb{K}: p^{-1}<d^{\prime}(x, F)\right\} & \text { if } m=0 .\end{cases}
$$

Now define the function $d(\cdot, F): \mathbb{K} \rightarrow \mathbb{Q}_{p}$ as follows:

$$
d(x, F)= \begin{cases}0 & \text { if } d^{\prime}(x, F)=0 \\ p^{m+1} & \text { si } x \in F_{m}\end{cases}
$$

Notice that $d(x, F)=0$ if and only if $x \in F$, and $|d(x, F)|_{p} \leq d^{\prime}(x, F)$ for every $x \in \mathbb{K}$.

For every $B \in \mathcal{B}$, define the functions $d_{B}(x)=\phi_{B}(x) d(x, F)$ for every $x \in \mathbb{K}$. We will prove that the family of functions $\left\{d_{B}: B \in \mathcal{B}\right\}$ are continuous only at the points belonging to $F$. Take $x \in F$, i.e., $d_{B}(x)=0$, and let $\left(x_{n}\right)_{n \geq 1} \subset \mathbb{K}$ be a sequence converging to $x$, then $\left(d_{B}\left(x_{n}\right)\right)_{n \geq 1}$ converges to 0 . Indeed, notice that for every $n \in \mathbb{N}$ we have

$$
0 \leq\left|d_{B}\left(x_{n}\right)\right|_{p}=\left|\phi_{B}\left(x_{n}\right) d\left(x_{n}, F\right)\right|_{p} \leq\left|d\left(x_{n}, F\right)\right|_{p} \leq d^{\prime}\left(x_{n}, F\right)
$$

If there are finitely many $n \in \mathbb{N}$ such that $d^{\prime}\left(x_{n}, F\right) \neq 0$, then clearly $\left(d_{B}\left(x_{n}\right)\right)_{n \geq 1}$ converges to 0 . If not, then the subsequence $\left(x_{n_{k}}\right)_{k \geq 1}$ of $\left(x_{n}\right)_{n \geq 1}$ with $d^{\prime}\left(x_{n_{k}}, F\right) \neq 0$ satisfies that $\left(d_{B}\left(x_{n_{k}}\right)\right)_{k \geq 1}$ converges to 0 since $\left(d^{\prime}\left(x_{n_{k}}, F\right)\right)_{k \geq 1}$ converges to 0 . The latter is true since $\left(x_{n_{k}}\right)_{k \geq 1}$ also converges to $x, d^{\prime}(\cdot, F)$ is continuous and $\left|d\left(x_{n}, F\right)\right|_{p} \leq d^{\prime}\left(x_{n}, F\right)$. Thus, $d_{B}\left\lceil F\right.$ is continuous. Now take $x \notin F$. By means of contradiction, assume that $d_{B}$ is continuous at $x$. We have two cases:

Case (1): Assume that $\phi_{B}(x)=0$. As $\phi_{B}$ is ES from $\mathbb{K}$ to $\mathbb{Z}_{p}$, we can choose a sequence $\left(x_{n}\right)_{n \geq 1} \subset F^{c}$ converging to $x$ such that $\phi_{B}\left(x_{n}\right)=1$ for every $n \in \mathbb{N}$. Since $\left(x_{n}\right)_{n \geq 1}$ converges to $x \in F_{m}$ for some $m \in \mathbb{N}_{0}$, we have that there exists $n_{0} \in \mathbb{N}$ 
such that $\left|d_{B}\left(x_{n}\right)\right|_{p} \geq p^{-m-1}$ for every $n \geq n_{0}$. Hence, $\left(d_{B}\left(x_{n}\right)\right)_{n \geq 1}$ cannot converge to 0 , a contradiction.

Case (2): Assume that $\phi_{B}(x) \neq 0$. Once again, as $\phi_{B}$ is ES from $\mathbb{K}$ to $\mathbb{Z}_{p}$, we can choose a sequence $\left(x_{n}\right)_{n \geq 1} \subset F^{c}$ converging to $x$ such that $\phi_{B}\left(x_{n}\right)=0$ for every $n \in \mathbb{N}$. Since $x \in F_{m}$ for some $m \in \mathbb{N}_{0}$, we have that $d_{B}(x) \neq 0$. But $d_{B}\left(x_{n}\right)=0$ for every $n \in \mathbb{N}$, a contradiction.

Thus $d_{B}$ is not continuous at the points outside $F$.

Let $B_{1} \in \mathcal{B}$, we will prove that $d_{B_{1}}$ does not belong to the algebra generated by $\left\{d_{B}: B \in \mathcal{B}\right\} \backslash\left\{d_{B_{1}}\right\}$. Assume that $d_{B_{1}}$ can be written as $P\left(d_{B_{2}}, \ldots, d_{B_{n}}\right)$, where $B_{2}, \ldots, B_{n} \in \mathcal{B} \backslash B_{1}$ are distinct and $P$ is a polynomial in $n-1$ variables with coefficients in $\mathbb{K} \backslash\{0\}$ and without free term.

Fix $x=\beta+y \in F^{c}$. There exists $\varepsilon>0$ such that $B(x, \varepsilon) \subseteq F^{c}$. Analogously to the proof of Corollary 3.2 we have that given $z \in B(\beta, \varepsilon) \cap \mathbb{Q}_{p}$, then $z+y \in B(x, \varepsilon)$. Take $\alpha \in B_{1} \cap B_{2}^{c} \cap \cdots \cap B_{n}^{c}$. Then, for every $\bar{x}=z+y \in F^{c}$ with $z \in B(\beta, \varepsilon) \cap \mathbb{Q}_{p} \cap A_{\alpha}$, we have

$$
\begin{aligned}
\varphi_{B_{1}}(z) d(\bar{x}, F) & =\phi_{B_{1}}(\bar{x}) d(\bar{x}, F)=d_{B_{1}}(\bar{x}) \\
& =P\left(d_{B_{2}}(\bar{x}), \ldots, d_{B_{n}}(\bar{x})\right)=P(0, \ldots, 0)=0 .
\end{aligned}
$$

But, since $\varphi_{B_{1}}\left[B(\beta, \varepsilon) \cap \mathbb{Q}_{p} \cap A_{\alpha}\right]=\mathbb{Z}_{p}$ and $d(x, F) \neq 0$ for every $x \in F^{c}$, we have a contradiction.

It is clear that any algebraic combination of the functions $\left\{d_{B}: B \in \mathcal{B}\right\}$ over $\mathbb{K}$ is continuous at the points belonging to $F$. It remains to show the following: Given $B_{1}, \ldots, B_{n} \in \mathcal{B}$ distinct and assuming that $P$ is a polynomial in $n$ variables with coefficients in $\mathbb{K} \backslash\{0\}$ and without free term such that $P\left(d_{B_{1}}\left(x_{0}\right), \ldots, d_{B_{n}}\left(x_{0}\right)\right) \neq 0$ for some $x_{0} \in \mathbb{K} \backslash\{0\}$, then $P\left(d_{B_{1}}, \ldots, d_{B_{n}}\right)$ is discontinuous outside $F$. Now, there exists $\alpha \in B_{1} \cup \cdots \cup B_{n}$ such that $x_{0}=\beta_{0}+y_{0}$ with $\beta_{0} \in A_{\alpha}$ and $y_{0} \in \operatorname{span}\langle\mathcal{H} \backslash\{1\}\rangle$, since otherwise $P\left(d_{B_{1}}\left(x_{0}\right), \ldots, d_{B_{n}}\left(x_{0}\right)\right)=P(0, \ldots, 0)=0$. Moreover, $x_{0} \in F^{c}$, if not, then $P\left(d_{B_{1}}\left(x_{0}\right), \ldots, d_{B_{n}}\left(x_{0}\right)\right)=P(0, \ldots, 0)=0$. Hence, for any $x=\beta+y \in F^{c}$ with $\beta \in A_{\alpha}$, we have that $P\left(d_{B_{1}}(x), \ldots, d_{B_{n}}(x)\right)$ is of the form $P_{1}\left(t_{\alpha}(\beta) d(x, F)\right)$, where $P_{1}$ is a polynomial in 1 variable with coefficients in $\mathbb{K} \backslash\{0\}$ and without free term. Fix $x \in F_{m}$ for some $m \in \mathbb{N}_{0}$. If $m \neq 0$, take $V_{x}$ a neighborhood of $x$ sufficiently small contained in $F_{m} \cup F_{m-1}$. If $m=0$, take $V_{x}$ contained in $F_{0}$. Notice that there exists a neighborhood $U_{\beta}$ of $\beta$ such that $U_{\beta}+y \subset V_{x}$. We have two cases:

Case (1): Assume that $P\left(d_{B_{1}}(x), \ldots, d_{B_{n}}(x)\right)=0$. Since $P_{1}$ takes the value 0 at most on a finite set $C \subset \mathbb{K}$, take a sequence $\left(x_{r}\right)_{r \geq 1}$ that converges to $x$ satisfying: $x_{r}=$ $\beta_{r}+y_{r} \in V_{x}$ with $\beta_{r} \in A_{\alpha} \cap U_{\beta}$ and $y_{r} \in \operatorname{span}\langle\mathcal{H} \backslash\{1\}\rangle,\left(t_{\alpha}\left(\beta_{r}\right)\right)_{r \geq 1}$ is a constant sequence, and $\left(t_{\alpha}\left(\beta_{r}\right) d\left(x_{r}, F\right)\right)_{n \geq 1} \not \subset \cup_{z \in C} B(z, \varepsilon)$ with $\varepsilon>0$ sufficiently small. This can be done as $t_{\alpha}$ is ES from $\mathbb{Q}_{p}$ to $\mathbb{Z}_{p}$ and $d\left(x_{r}, F\right) \in\left\{p^{m}, p^{m+1}\right\}$ for every $r \in \mathbb{N}$. Hence, $\left(P_{1}\left(t_{\alpha}\left(\beta_{r}\right) d\left(x_{r}, F\right)\right)\right)_{r \geq 1}$ does not converge to 0 .

Case (2): Assume that $P\left(d_{B_{1}}(x), \ldots, d_{B_{n}}(x)\right) \neq 0$. Take $\alpha^{\prime} \in B_{1}^{c} \cap \cdots \cap B_{n}^{c}$ and a sequence $\left(x_{r}\right)_{r \geq 1}$ converging to $x$ such that $x_{r}=\beta_{r}+y_{r} \in V_{x}$ with $\beta_{r} \in A_{\alpha^{\prime}}$ and $y_{r} \in \operatorname{span}\langle\mathcal{H} \backslash\{1\}\rangle$. Now we have that $\left(P\left(d_{B_{1}}\left(x_{r}\right), \ldots, d_{B_{n}}\left(x_{r}\right)\right)\right)_{r \geq 1}$ is the zero sequence which cannot converge to $P\left(d_{B_{1}}(x), \ldots, d_{B_{n}}(x)\right)$.

Thus, $P\left(d_{B_{1}}, \ldots, d_{B_{n}}\right)$ is not continuous at any point of $F^{c}$.

In the next result, we give a $p$-adic analogous of [3, theorem 5.1]. Here we need a more delicate argument in comparison to the real case. We equip the space $\mathbb{Q}_{p}^{n}$ with the following 
norm:

$$
|x|_{p}=\max _{1 \leq i \leq n}\left|x_{i}\right|_{p}, \quad x=\left(x_{1}, \ldots, x_{n}\right) \in \mathbb{Q}_{p}^{n} .
$$

Theorem 3.7 Let $n \geq 2$ and $x_{0} \in \mathbb{Q}_{p}^{n}$ with $p>2$. The family of separately continuous functions $\mathbb{Q}_{p}^{n} \rightarrow \mathbb{Q}_{p}$ that are everywhere continuous except at $x_{0}$ is $\mathfrak{c}$-lineable.

Proof We will prove the result in the case when $n=2$. Fix $x_{0}=\mathbf{0}$, where $\mathbf{0}:=(0,0)$. For any $x, y \in \mathbb{Z}_{p}$ written in the canonical form $x=\sum_{n=0}^{\infty} a_{n} p^{n}$ and $y=\sum_{n=0}^{\infty} b_{n} p^{n}$, let us denote $c(x, y):=\sum_{n=0}^{\infty} c_{n} p^{n}$, where $c_{n}=a_{n}$ and $c_{2 n+1}=b_{n}$. Let us also denote $\bar{x}=\sum_{n=1}^{\infty}\left(a_{2 n+1} p^{2 n}+a_{2 n} p^{2 n+1}\right)$ for any $x=\sum_{n=0}^{\infty} a_{n} p^{n} \in \mathbb{Z}_{p}$.

Let us define $f: \mathbb{Q}_{p} \times \mathbb{Q}_{p} \rightarrow \mathbb{Q}_{p}$ as follows

$$
f(x, y)= \begin{cases}\frac{c(x, y) c(\bar{y}, \bar{x})}{c(y, x) c(\bar{x}, \bar{y})} & \text { if }(x, y) \in \mathbb{Z}_{p}^{2} \backslash\{\mathbf{0}\}, \\ 1 & \text { otherwise. }\end{cases}
$$

Notice that if $(x, y) \notin \mathbb{Z}_{p}^{2} \backslash\{\boldsymbol{0}\}$, then there exists a neighborhood $V_{(x, y)}$ of $(x, y)$ such that $f \uparrow V_{(x, y)} \equiv 1$. Indeed, as $(x, y) \notin \mathbb{Z}_{p}^{2} \backslash\{\boldsymbol{0}\}$, there exists $r \in \mathbb{N}$ such that $|(x, y)|_{p}=p^{r}$. Hence, $(x, y) \in S\left(\mathbf{0}, p^{r}\right)$ which is an open set and, therefore, for every $0<\varepsilon<p^{r}$, we have $B((x, y), \varepsilon) \subset S\left(\mathbf{0}, p^{r}\right)$. Thus, $f\lceil B((x, y), \varepsilon) \equiv 1$.

Now, on the one hand, for any $\left(x^{\prime}, y^{\prime}\right) \in \mathbb{Z}_{p}^{2} \backslash\{\boldsymbol{0}\}$, there exists $(x, y) \in \mathbb{Z}_{p}^{2} \backslash\{\boldsymbol{0}\}$ such that $\left|x-x^{\prime}\right|<p^{-2 r+1}$ and $\left|y-y^{\prime}\right|<p^{-2 r+1}$ with $r$ sufficiently large. Then,

$$
\begin{aligned}
& \frac{c\left(x^{\prime}, y^{\prime}\right)}{c\left(y^{\prime}, x^{\prime}\right)}=\frac{a_{0}^{\prime}+b_{0}^{\prime} p+\cdots+b_{2 r-1}^{\prime} p^{4 r-1}+a_{2 r}^{\prime} p^{4 r}+\cdots}{b_{0}^{\prime}+a_{0}^{\prime} p+\cdots+a_{2 r-1}^{\prime} p^{4 r-1}+b_{2 r}^{\prime} p^{4 r}+\cdots}, \\
& \frac{c\left(\overline{y^{\prime}}, \overline{x^{\prime}}\right)}{c\left(\overline{x^{\prime}}, \overline{y^{\prime}}\right)}=\frac{b_{1}^{\prime}+a_{1}^{\prime} p+b_{0}^{\prime} p^{2}+a_{0}^{\prime} p^{3}+\cdots+a_{2 r-2}^{\prime} p^{4 r-1}+d_{2 r} p^{4 r}+\cdots}{a_{1}^{\prime}+b_{1}^{\prime} p+a_{0}^{\prime} p^{2}+b_{0}^{\prime} p^{3}+\cdots+b_{2 r-2}^{\prime} p^{4 r-1}+c_{2 r} p^{4 r}+\cdots} .
\end{aligned}
$$

Notice that we have the following

$$
\left|f\left(x^{\prime}, y^{\prime}\right)-f(x, y)\right|_{p} \leq \frac{p^{-4 r}}{\left|c\left(y^{\prime}, x^{\prime}\right)\right|_{p}\left|c\left(\overline{x^{\prime}}, \overline{y^{\prime}}\right)\right|_{p}|c(y, x)|_{p}|c(\bar{x}, \bar{y})|_{p}} \longrightarrow 0,
$$

as $r \rightarrow \infty$.

On the other hand, for any $k \in \mathbb{N}$, consider the sequence $\left(x_{n}^{k}, y_{n}^{k}\right)_{n \geq 0} \subset \mathbb{Z}_{p}^{2}$, where $x_{n}^{k}=2 p^{2 n}+2 p^{2 n+1}+2 p^{2 n+2 k+1}$ and $y_{n}^{k}=p^{2 n}+p^{2 n+1}+p^{2 n+2 k+1}$ for every $n \geq 0$. Then, for any $n \geq 0$, we have that

$$
\begin{aligned}
f\left(x_{n}^{k}, y_{n}^{k}\right)= & \frac{2+p+2 p^{2}+p^{3}+2 p^{4 k+2}+p^{4 k+3}}{1+2 p+p^{2}+2 p^{3}+p^{4 k+2}+2 p^{4 k+3}} \\
& \cdot \frac{1+2 p+p^{2}+2 p^{3}+p^{4 k}+2 p^{4 k+1}}{2+p+2 p^{2}+p^{3}+2 p^{4 k}+p^{4 k+1}} .
\end{aligned}
$$

Thus, for any $k \in \mathbb{N}$, when $n \rightarrow \infty$ we have that $\left(x_{n}^{k}, y_{n}^{k}\right)_{n \geq 0}$ tends to zero in $\mathbb{Q}_{p}^{2}$ and $f\left(x_{n}^{k}, y_{n}^{k}\right)$ tends in $\mathbb{Q}_{p}$ to the constant

$$
\beta_{k}=\frac{2+p+2 p^{2}+p^{3}+2 p^{4 k+2}+p^{4 k+3}}{1+2 p+p^{2}+2 p^{3}+p^{4 k+2}+2 p^{4 k+3}} \cdot \frac{1+2 p+p^{2}+2 p^{3}+p^{4 k}+2 p^{4 k+1}}{2+p+2 p^{2}+p^{3}+2 p^{4 k}+p^{4 k+1}} .
$$


Notice that for distinct $k, k^{\prime} \in \mathbb{N}$, the $p$-adic numbers $\beta_{k}, \beta_{k^{\prime}}$ are $p$-integers and also distinct. Hence $f$ is not continuous at $\mathbf{0}$. It is easy to see that $f$ is separately continuous since $f(0, x)=$ $f(y, 0)=1$ for every $x, y \in \mathbb{Q}_{p}$.

Let $\mathcal{V}$ be a family of c-many analytic linearly independent functions from $\mathbb{Q}_{p}$ to $\mathbb{Q}_{p}$. For every $v \in \mathcal{V}$, let us define the function $f_{v}=v \circ f: \mathbb{Q}_{p}^{2} \rightarrow \mathbb{Q}_{p}$. Take $F=\sum_{j=1}^{n} \alpha_{j} f_{v_{j}}$, where $f_{v_{j}}$ are distinct and $\alpha_{j} \in \mathbb{Q}_{p}$ for any $1 \leq j \leq n$. If $F$ were identically zero, then $\sum_{j=1}^{n} \alpha_{j} v_{j}\left(\beta_{k}\right)=0$ for every $k \in \mathbb{N}$. Therefore, the function $\sum_{j=1}^{n} \alpha_{j} v_{j}$ (which is analytic) would be equal to zero on an infinite set which contradicts Strassman's theorem [30, theorem 4.4.6]. Notice that by construction the function $F$ is continuous except at $\mathbf{0}$ and also separately continuous.

The next result shows that the inclusion

$$
\operatorname{Lip}_{1 /(m-1)}\left(\mathbb{K}_{1} \rightarrow \mathbb{K}_{2}\right) \subset \operatorname{Lip}_{1 / m}\left(\mathbb{K}_{1} \rightarrow \mathbb{K}_{2}\right)
$$

is proper, with $\mathbb{K}_{1} \in\left\{\mathbb{Z}_{p}, \mathbb{Q}_{p}\right\}$ and $\mathbb{K}_{2} \in\left\{\mathbb{Q}_{p}, \overline{\mathbb{Q}_{p}}, \mathbb{C}_{p}, \Omega_{p}\right\}$; and large enough to contain a $\mathfrak{c}$-dimensional linear space over $\mathbb{K}_{2}$.

Theorem 3.8 For every $\mathbb{K}_{1} \in\left\{\mathbb{Z}_{p}, \mathbb{Q}_{p}\right\}, \mathbb{K}_{2} \in\left\{\mathbb{Q}_{p}, \overline{\mathbb{Q}_{p}}, \mathbb{C}_{p}, \Omega_{p}\right\}$ and any integer $m \geq 2$, the set $\operatorname{Lip}_{1 / m}\left(\mathbb{K}_{1} \rightarrow \mathbb{K}_{2}\right) \backslash \operatorname{Lip}_{1 /(m-1)}\left(\mathbb{K}_{1} \rightarrow \mathbb{K}_{2}\right)$ is c-lineable (as a $\mathbb{K}_{2}$-vector space).

Proof Fix $\mathbb{K}_{1} \in\left\{\mathbb{Z}_{p}, \mathbb{Q}_{p}\right\}, \mathbb{K}_{2} \in\left\{\mathbb{Q}_{p}, \overline{\mathbb{Q}_{p}}, \mathbb{C}_{p}, \Omega_{p}\right\}$ and an integer $m \geq 2$. Given $q \in \mathbb{P}$ we will define a function $f_{q}: \mathbb{K}_{1} \rightarrow \mathbb{Z}_{p}$ in the following way: for every $x=\sum_{n=r}^{\infty} a_{n} p^{n}$, with $r \in \mathbb{Z}$, take $f_{q}(x)=\sum_{n=0}^{\infty} a_{m(m-1) q^{n+1}} p^{(m-1) q^{n+1}}$.

Let us prove that for any $q \in \mathbb{P}$, we have $f_{q} \in \operatorname{Lip}_{1 / m}\left(\mathbb{K}_{1} \rightarrow \mathbb{K}_{2}\right)$. Take $x, y \in \mathbb{K}_{1}$. Clearly $\left|f_{q}(x)-f_{q}(y)\right|_{p} \leq|x-y|_{p}^{1 / m}$ when $x=y$, so assume that $x \neq y$. Hence there exists $t \in \mathbb{Z}$ such that $|x-y|_{p}=p^{-t}$. We will divide the proof into two cases:

Case (1): If $t \leq m(m-1) q$, then $\left|f_{q}(x)-f_{q}(y)\right|_{p} \leq p^{-(m-1) q}$. Thus $\left|f_{q}(x)-f_{q}(y)\right|_{p} \leq$ $p^{-(m-1) q} \leq p^{-t / m}=|x-y|_{p}^{1 / m}$.

Case (2): If $t>m(m-1) q$, then there exists $n_{t} \in \mathbb{N}_{0}$ such that $m(m-1) q^{n_{t}+1}<t \leq$ $m(m-1) q^{n_{t}+2}$. Therefore,

$$
\left|f_{q}(x)-f_{q}(y)\right|_{p} \leq p^{-(m-1) q^{n_{t}+2}},
$$

and, hence, we have

$$
\left|f_{q}(x)-f_{q}(y)\right|_{p} \leq p^{-(m-1) q^{n_{t}+2}} \leq p^{-t / m}=|x-y|_{p}^{1 / m} .
$$

For every $P \in \mathcal{P}$, let us define $F_{P}=\sum_{q \in P} f_{q}$. Fix $P \in \mathcal{P}$. The function $F_{P}$ is well defined since, for every $x \in \mathbb{K}_{1}, F_{P}(x)$ exists. Indeed, it is enough to prove that $\left(f_{q}(x)\right)_{q \in P}$ converges to 0 for every $x \in \mathbb{K}_{1}$. Take $x \in \mathbb{K}_{1}$, then $\left|f_{q}(x)\right|_{p} \leq p^{-(m-1) q} \rightarrow 0$ as $q \rightarrow \infty$.

Let us prove now that $F_{P} \notin \operatorname{Lip}_{1 /(m-1)}\left(\mathbb{K}_{1} \rightarrow \mathbb{K}_{2}\right)$ for every $P \in \mathcal{P}$. Fix $P \in \mathcal{P}$. For every $q \in P$, take $x=0$ and $y_{i}=p^{m(m-1) q^{i+1}}$ for any $i \in \mathbb{N}_{0}$. Notice that $\left|y_{i}\right|_{p}=p^{-m(m-1) q^{i+1}}$ and $\left|F_{P}\left(y_{i}\right)\right|=p^{-(m-1) q^{i+1}}$ for any $i \in \mathbb{N}_{0}$. Assume that $F_{P} \in \operatorname{Lip}_{1 /(m-1)}\left(\mathbb{K}_{1} \rightarrow \mathbb{K}_{2}\right)$, then there exists $M>0$ such that $\left|F_{P}(x)-F_{P}\left(y_{i}\right)\right|_{p} \leq M\left|x-y_{i}\right|_{p}^{1 /(m-1)}$ for any $i \in \mathbb{N}_{0}$. Thus, we have $p^{-(m-1) q^{i+1}} \leq M p^{-m q^{i+1}}$ for any $i \in \mathbb{N}_{0}$, that is, $p^{q^{i+1}} \leq M$ for any $i \in \mathbb{N}_{0}$, which is absurd. 
Now we will show that $F_{P} \in \operatorname{Lip}_{1 / m}\left(\mathbb{K}_{1} \rightarrow \mathbb{K}_{2}\right)$ for every $P \in \mathcal{P}$. For every $x, y \in \mathbb{K}_{1}$ we have that

$$
\left|F_{P}(x)-F_{P}(y)\right|_{p}=\left|\sum_{q \in P}\left(f_{q}(x)-f_{q}(y)\right)\right|_{p} \leq \sup _{q \in P}\left|f_{q}(x)-f_{q}(y)\right|_{p} \leq|x-y|_{p}^{1 / m} .
$$

Let us show that the functions in the set $\left\{F_{P}: P \in \mathcal{P}\right\}$ are linearly independent over $\mathbb{K}_{2}$. Take $n$ distinct elements $P_{1}, \ldots, P_{n}$ of $\mathcal{P}$ and consider $F=\sum_{j=1}^{n} b_{j} F_{P_{j}}$ with $b_{j} \in \mathbb{K}_{2} \backslash\{0\}$ for every $1 \leq j \leq n$. Notice that by picking $q \in P_{1} \cap P_{2}^{c} \cap \cdots \cap P_{n}^{c}$ and $x=p^{m(m-1) q}$, we have $F(x)=b_{1} p^{(m-1) q}$. If $F$ were the identically zero function, then $b_{1}=0$ and we have a contradiction.

Since $\operatorname{Lip}_{1 / m}\left(\mathbb{K}_{1} \rightarrow \mathbb{K}_{2}\right)$ forms a vector space over $\mathbb{K}_{2}$, we clearly have that any linear combination over $\mathbb{K}_{2}$ of the functions in $\left\{F_{P}: P \in \mathcal{P}\right\}$ belongs to $\operatorname{Lip}_{1 / m}\left(\mathbb{K}_{1} \rightarrow \mathbb{K}_{2}\right)$. It remains to prove that given $F=\sum_{j=1}^{n} b_{j} F_{P_{j}}$ with $b_{j} \in \mathbb{K}_{2} \backslash\{0\}$ and $P_{1}, \ldots, P_{n}$ distinct elements of $\mathcal{P}$, we have that $F \notin \operatorname{Lip}_{1 /(m-1)}\left(\mathbb{K}_{1} \rightarrow \mathbb{K}_{2}\right)$. Take $q \in P_{1} \cap P_{2}^{c} \cap \cdots \cap P_{n}^{c}, x=0$ and $y_{i}=p^{m(m-1) q^{i+1}}$ for any $i \in \mathbb{N}_{0}$. Notice that $\left|F(x)-F\left(y_{i}\right)\right|_{p}=\left|b_{1}\right| p^{-(m-1) q^{i+1}}$ for any $i \in \mathbb{N}_{0}$. By using the same arguments as above we have that $F \notin \operatorname{Lip}_{1 /(m-1)}\left(\mathbb{K}_{1} \rightarrow \mathbb{K}_{2}\right)$.

In [37, theorem 4.5], the authors prove that the family of uniformly continuous functions $\mathbb{Z}_{p} \rightarrow \mathbb{Q}_{p}$ that are nowhere differentiable functions is c-lineable provided that $p>2$. These functions can be named Dieudonné's monster in parallel to its counterpart in the real case named Weierstrass' monster.

By taking $m=2$ in Theorem 3.8, note that the functions in the set $\operatorname{Lip}_{1 / 2}\left(\mathbb{K}_{1} \rightarrow\right.$ $\left.\mathbb{K}_{2}\right) \backslash \operatorname{Lip}_{1}\left(\mathbb{K}_{1} \rightarrow \mathbb{K}_{2}\right)$, with $\mathbb{K}_{1} \in\left\{\mathbb{Q}_{p}, \overline{\mathbb{Q}_{p}}\right\}$ and $\mathbb{K}_{2} \in\left\{\mathbb{Q}_{p}, \overline{\mathbb{Q}_{p}}, \mathbb{C}_{p}, \Omega_{p}\right\}$, are uniformly continuous and nowhere differentiable. Hence, we have the following result.

Corollary 3.9 For every $\mathbb{K}_{1} \in\left\{\mathbb{Z}_{p}, \mathbb{Q}_{p}\right\}$ and $\mathbb{K}_{2} \in\left\{\mathbb{Q}_{p}, \overline{\mathbb{Q}_{p}}, \mathbb{C}_{p}, \Omega_{p}\right\}$, the family of uniformly continuous nowhere differentiable functions $\mathbb{K}_{1} \rightarrow \mathbb{K}_{2}$ is c-lineable (as a $\mathbb{K}_{2}$-vector space).

By an antiderivative of a function $f$ we mean any function $F$ such that $F^{\prime}=f$. We give a weaker corrected version of [38, theorem 2.7] . Let us remark that the functions constructed in its proof are not everywhere discontinuous and do not generate an algebra.

Proposition 3.10 The family of discontinuous functions $\mathbb{Q}_{p} \rightarrow \mathbb{Q}_{p}$ with finite range that have antiderivative is $\mathfrak{c}$-lineable.

Proof For every $N \in \mathcal{N}$, let us define $g_{N}: \mathbb{Q}_{p} \rightarrow \mathbb{Q}_{p}$ as

$$
g_{N}(x)= \begin{cases}0 & \text { if }|x|_{p}=p^{-n} \text { with } n \in N \\ 1 & \text { otherwise. }\end{cases}
$$

For any $N \in \mathcal{N}$, the function $g_{N}$ clearly has finite range and has an antiderivative $G_{N}$ given by

$$
G_{N}(x)= \begin{cases}x_{n} p^{n}+\cdots+x_{2 n} p^{2 n} & \text { if }|x|_{p}=p^{-n} \text { with } n \in N \text { and } x=\sum_{i=1}^{\infty} x_{i} p^{i}, \\ x & \text { otherwise. }\end{cases}
$$

Firstly, if $x \neq 0$ and $|x|_{p} \notin\left\{p^{-n}: n \in N\right\}$, there is a neighborhood $U_{x}$ of $x$ such that $G_{N}\left\lceil U_{x}\right.$ is the identity function. Thus, $G_{N}$ is differentiable at $x$ and the derivative is $1=g_{N}(x)$. Secondly, if $|x|_{p} \in\left\{p^{-n}: n \in N\right\}$, there is a neighborhood $U_{x}$ of $x$ such that 
$G_{N}\left\lceil U_{x}\right.$ is constant. Hence, $G_{N}$ is differentiable at $x$ and the derivative is $0=g_{N}(x)$. Lastly, we will analyze the case when $x=0$. Notice that

$$
\frac{G_{N}(x)-G_{N}(0)}{x-0}= \begin{cases}1-\frac{\sum_{i=2 n+1}^{\infty} x_{i} p^{i}}{x_{n} p^{n}+\sum_{i=n+1}^{\infty} x_{i} p^{i}} & \text { if }|x|_{p}=p^{-n} \text { with } n \in N \\ & \text { and } x=\sum_{i=1}^{\infty} x_{i} p^{i}, \\ 1 & \text { otherwise. }\end{cases}
$$

To see that $G_{N}$ is differentiable at 0 and $G_{N}^{\prime}(0)=1=g_{N}(0)$, it is enough to prove in (3.2) that

$$
\lim _{n \rightarrow \infty} \frac{\sum_{i=2 n+1}^{\infty} x_{i} p^{i}}{x_{n} p^{n}+\sum_{i=n+1}^{\infty} x_{i} p^{i}}=0 .
$$

But the latter simply follows from the fact that

$$
\left|\frac{\sum_{i=2 n+1}^{\infty} x_{i} p^{i}}{x_{n} p^{n}+\sum_{i=n+1}^{\infty} x_{i} p^{i}}\right|_{p}=\left|\sum_{i=2 n+1}^{\infty} x_{i} p^{i}\right|_{p} p^{n}<p^{n-2 n-1}=p^{-(n+1)} \rightarrow 0,
$$

as $n \rightarrow \infty$.

Moreover, $g_{N}$ is discontinuous at 0 . Indeed, fix $\varepsilon=p^{-1}$. For every $n \in N$ and every $x \in \mathbb{N}$ with $|x|_{p}=p^{-n}$, we have $\left|g_{N}(0)-g_{N}(x)\right|_{p}=1>p^{-1}$. Obviously $\alpha g_{N}$ has finite range, is discontinuous at 0 and has antiderivative (i.e., $\alpha G_{n}^{\prime}=\alpha g_{N}$ ), for every $\alpha \in \mathbb{Q}_{p} \backslash\{0\}$ and $N \in \mathcal{N}$

Now, take $\alpha_{1}, \ldots, \alpha_{k} \in \mathbb{Q}_{p}$ and $g_{N_{1}}, \ldots, g_{N_{k}}$ distinct, where $k \in \mathbb{N}$. Define $g=\alpha_{1} g_{N_{1}}+$ $\cdots+\alpha_{k} g_{N_{k}}$, and assume first that $g \equiv 0$. Then, for any $n \in N_{1}^{c} \cap N_{2} \cap \cdots \cap N_{k}$ and any $x \in \mathbb{Q}_{p}$ with $|x|_{p}=p^{-n}$, we have $0=g(x)=\alpha_{1}$. Repeating the same argument we have that $\alpha_{i}=0$ for every $i \in\{1, \ldots, k\}$. Assume second that $\alpha_{i} \neq 0$ for every $i \in\{1, \ldots, k\}$. Then $g$ clearly has finite range and $\alpha_{1} G_{1}+\cdots+\alpha_{k} G_{k}$ is an antiderivative. It remains to prove that $g$ is discontinuous at 0 . Assume that $\left|\alpha_{1}\right|_{p}=p^{k}$, where $k \in \mathbb{Z}$. Fix $\varepsilon=p^{k-1}$. For every $n \in N_{1} \cap N_{2}^{c} \cap \cdots \cap N_{k}^{c}$ and every $x \in \mathbb{Q}_{p}$ with $|x|_{p}=p^{-n}$, we have $|g(0)-g(x)|_{p}=\left|\alpha_{1}+\cdots+\alpha_{k}-\left(\alpha_{2}+\cdots+\alpha_{k}\right)\right|_{p}=\left|\alpha_{1}\right|_{p}>p^{k-1}$.

\section{Lineability of sets of $p$-adic differentiable and analytic functions}

We commence by showing the failure of celebrated Liouville's theorem on $p$-adic numbers field. Let us remark that Liouville's theorem, which states that a bounded analytical function of a field $\mathbb{K}$ is constant, holds true in any not locally compact complete non-trivially valued field [44, theorem 42.6]. But this is not true for the locally compact case as illustrated in [44, example 43.1]. We show that this is a generic algebraic behavior of bounded analytic functions on $\mathbb{Q}_{p}$.

Theorem 4.1 The family of non-constant bounded analytic functions $\mathbb{Q}_{p} \rightarrow \mathbb{Q}_{p}$ is c-lineable.

Proof First, it is important to mention that the function $f$ given in the proof of [44, example 43.1] satisfies $f(0)=1$. Also, by the proof of [44, example 43.1], we can assume that $|f(x)|_{p} \leq p^{-1}$. By considering now $\tilde{f}=f-1$, we have a non-constant bounded analytic function from $\mathbb{Q}_{p}$ to $\mathbb{Q}_{p}$, which will be called again $f$ for simplicity, that satisfies the following properties: $f(0)=0$ and $|f(x)|_{p} \leq 1$ for any $x \in \mathbb{Q}_{p}$. Hence, since $f$ is analytic, there exists $\left(a_{n}\right)_{n \geq 0} \subset \mathbb{Q}_{p}$ such that $f(x)=\sum_{i=0}^{\infty} a_{i} x^{i}$ for any $x \in \mathbb{Q}_{p}$ and, as $f(0)=0$, notice that $a_{0}=0$. 
Given $q \in \mathbb{P}$, let us define the function $g_{q}(x)=p^{q} f(x)$ for every $x \in \mathbb{Q}_{p}$. For every $P \in \mathcal{P}$, take $G_{P}=\sum_{q \in P} g_{q}$. Fix $P \in \mathcal{P}$. The function $G_{P}$ is well defined since $G_{P}(x)$ exists for every $x \in \mathbb{Q}_{p}$. Indeed, for every $x \in \mathbb{Q}_{p}$, we have that $\left(g_{q}(x)\right)_{q \in P}$ converges to 0 since $\left|g_{q}(x)\right|_{p} \leq p^{-q} \rightarrow 0$ as $q \rightarrow \infty$. Moreover, the functions $G_{P}$ are bounded since for every $x \in \mathbb{Q}_{p}$ we have

$$
\left|G_{P}(x)\right|_{p}=\left|\sum_{q \in P} g_{p}(x)\right|_{p} \leq \sup _{q \in P}\left|g_{p}(x)\right|_{p} \leq 1 .
$$

Now we will prove that the functions $G_{P}$ are analytic in $\mathbb{Q}_{p}$. For every $\alpha \in \mathbb{Q} \backslash \mathbb{Z}$, let us define $a_{\alpha}=0$. Notice that the function $\sum_{\substack{i \in \mathbb{N} \\ q \in P}} p^{q} a_{\frac{i}{q}} x^{\frac{i}{q}}$ is clearly analytic in $\mathbb{Q}_{p}$ and for any integer $n \geq 2$ and every $x \in \mathbb{Q}_{p}$ we have

$$
\begin{aligned}
\left|\sum_{\substack{i \in \mathbb{N} \\
q \in P}} p^{q} a_{\frac{i}{q}} x^{\frac{i}{q}}-\sum_{q \in P} g_{p}(x)\right|_{q \leq n} & \leq \sup _{i \in \mathbb{N}}\left|\sum_{q \in P} p^{q} a_{\frac{i}{q}} x^{\frac{i}{q}}\right| \leq \sup _{i \in \mathbb{N}} \sup _{q \in P, q>n}\left|p^{q}\right|_{p}\left|a_{\frac{i}{q}} x^{\frac{i}{q}}\right|_{p} \\
& \leq \sup _{i \in \mathbb{N}} \sup _{q \in P, q>n}\left|p^{-(n+1)}\right|_{p}\left|a_{\frac{i}{q}} x^{\frac{i}{q}}\right|_{p}
\end{aligned}
$$

On the other hand, as $\sum_{i=0}^{\infty} a_{i} y^{i}$ converges for any $y \in \mathbb{Q}_{p}$, the sequence $\left(a_{i} y^{i}\right)_{i \geq 0}$ converges to 0 for any $y \in \mathbb{Q}_{p}$ (see, for instance, [35, proposition 3.3]). Hence, there exists $A_{y}>0$ such that $\left|a_{i} y^{i}\right|_{p} \leq A_{y}$ for any $i \in \mathbb{N}_{0}$. Thus

$$
\sup _{q \in P, q>n}\left|p^{-(n+1)}\right|_{p}\left|a_{\frac{i}{q}} x^{\frac{i}{q}}\right|_{p} \leq A_{x} p^{-(n+1)}
$$

for any $i \in \mathbb{N}_{0}$. Since the latter upper bound does not depend on $i$, we have

$$
\left|\sum_{\substack{i \in \mathbb{N} \\ q \in P}} p^{q} a_{\frac{i}{q}} x^{\frac{i}{q}}-\sum_{\substack{q \in P \\ q \leq n}} g_{p}(x)\right|_{p}=O\left(p^{-n}\right) .
$$

Hence $G_{P}(x)=\sum_{q \in P} g_{p}(x)=\sum_{\substack{i \in \mathbb{N} \\ q \in P}} p^{q} a_{\frac{i}{q}} x^{\frac{i}{q}}$ for every $x \in \mathbb{Q}_{p}$.

It is clear that any non-zero linear combination on $\mathbb{Q}_{p}$ of functions in the set $\left\{G_{P}: P \in \mathcal{P}\right\}$ is a non-constant, bounded and analytic function from $\mathbb{Q}_{p}$ to $\mathbb{Q}_{p}$. It remains to prove that they are linearly independent over $\mathbb{Q}_{p}$. Let $i_{0} \in \mathbb{N}$ be the index such that $a_{i_{0}} \neq 0$ and $a_{i}=0$ for every $0 \leq i \leq i_{0}-1$. Take $n$ distinct sets $P_{1}, \ldots, P_{n}$ in $\mathcal{P}$ and the function $G=\sum_{j=1}^{n} b_{j} G_{P_{j}}$ with $b_{j} \in \mathbb{Q}_{p} \backslash\{0\}$. Notice that by taking $q \in P_{1} \cap P_{2}^{c} \cap \cdots \cap P_{n}^{c}$, the coefficient of $x^{\frac{i}{q}}$ with $i=i_{0} q$ in the power series expansion of $G_{P}$ is $b_{1} p^{q} a_{i_{0}}$. Hence, if $G$ were identically zero, we would have $b_{1}=0$, which is absurd.

In the next proposition we study the failure of one of the standard results in real analysis about the interchange of limit and derivative. More specifically, as K. Mahler put it [40]: if a series $f(x)=\sum_{n} f_{n}(x)$ converges and the derived series $g(x)=\sum_{n} f_{n}^{\prime}(x)$ converges uniformly, $g(x)$ still need not be the derivative of $f(x)$. To do that, we need the van der Put expansion of a continuous function on $\mathbb{Z}_{p}$. For an integer $m>0$ and $x \in \mathbb{Z}_{p}$ define

$$
\psi_{m}(x) \equiv \text { characteristic function of the ball } B\left(m, p^{\left.-\left\lfloor\log _{p}(m)\right\rfloor\right]-1}\right)
$$


and

$$
\psi_{0}(x) \equiv \text { characteristic function of the ball } B(0,1 / p) .
$$

It is a well known result of van der Put that every continuous function $f: \mathbb{Z}_{p} \rightarrow \mathbb{K}$, where $\mathbb{K}$ is a complete extension of $\mathbb{Q}_{p}$, is represented by the series $f(x)=\sum_{m=0}^{\infty} \alpha_{m} \psi_{m}(x)$, where $\alpha_{m} \in \mathbb{K}$; see $[41,43,44]$. It should be noted that the series converges uniformly, and the functions $\psi_{m}$ form a basis of the space of locally constant functions $\mathbb{Z}_{p} \rightarrow \mathbb{K}$ (see [43, pp. 179-182]).

Now we are ready to prove our next result.

Theorem 4.2 For every $\mathbb{K} \in\left\{\mathbb{Q}_{p}, \overline{\mathbb{Q}_{p}}, \mathbb{C}_{p}, \Omega_{p}\right\}$, the set of functions $f: \mathbb{Z}_{p} \rightarrow \mathbb{K}$ such that there exists a sequence of differentiable functions $\left(f_{n}\right)_{n \geq 1}$ with $f_{n}: \mathbb{Z}_{p} \rightarrow \mathbb{K}, f_{n} \rightarrow f$ uniformly and $f_{n}^{\prime} \rightarrow g$ uniformly but $f^{\prime} \neq g$ is c-lineable.

Proof Fix $\mathbb{K} \in\left\{\mathbb{Q}_{p}, \overline{\mathbb{Q}_{p}}, \mathbb{C}_{p}, \Omega_{p}\right\}$. For every $N \in \mathcal{N}$, let us define the power series $f_{N}(x)=$ $\sum_{n \in N} p^{n^{2}} x^{n}$ for any $x \in \mathbb{Z}_{p}$. Clearly, the radius of convergence of $f_{N}$ is infinite for any $N \in \mathcal{N}$. Now, let $f=a_{1} f_{N_{1}}+\cdots+a_{k} f_{N_{k}}$, where $k \in \mathbb{N}, N_{1}, \ldots, N_{k} \in \mathcal{N}$ are distinct and $a_{1}, \ldots, a_{k} \in \mathbb{K} \backslash\{0\}$. Notice that $f$ is a power series with coefficients in $\mathbb{K}$. If $f$ were the zero power series, then the coefficients of $f$ in the terms $x^{n}$ with $n \in N_{1} \cap N_{2}^{c} \cap \cdots \cap N_{k}^{c}$, which are $a_{1} p^{n^{2}}$, would be zero. Thus, we would have $a_{1}=0$ which is a contradiction. An analogous approach shows that the derivative of $f$ given by $f^{\prime}=a_{1} f_{N_{1}}^{\prime}+\cdots+a_{k} f_{N_{k}}^{\prime}$, where $f_{N_{i}}^{\prime}(x)=\sum_{n \in N_{i}} n p^{n^{2}} x^{n-1}$, is not the zero power series.

Let $\mathcal{F}=\left\{f_{N}: N \in \mathcal{N}\right\}$. For any $f_{N} \in \mathcal{F}$ and $n \in \mathbb{N}_{0}$, take $f_{n, N}$ to be the partial sum $\sum_{i=0}^{n} \alpha_{i} \psi_{i}$ of the van der Put expansion of the function $f_{N}$. On the one hand, as noted above, the sequence $\left(f_{n, N}\right)_{n \geq 0}$ converges uniformly to $f_{N}$ for any $N \in \mathcal{N}$. On the other hand, in view of the fact that $\left(\psi_{i}\right)_{i \geq 0}$ is a basis of the space of locally constant functions $\mathbb{Z}_{p} \rightarrow \mathbb{K}$, we have that $f_{n, N}^{\prime} \equiv 0$ for any $n \in \mathbb{N}_{0}$ and $N \in \mathcal{N}$. Hence, $\left(f_{n, N}^{\prime}\right)_{n \geq 0}$ converges uniformly to the zero function for any $N \in \mathcal{N}$. But $f_{N}^{\prime} \not \equiv 0$ for any $N \in \mathcal{N}$. The same can be applied for any non-zero linear combination over $\mathbb{K}$ of the functions in $\mathcal{F}$.

It is well known that if $f: \mathbb{R} \rightarrow \mathbb{R}$ is differentiable with $f^{\prime} \equiv 0$, then $f$ is constant. This is not true in general in the $p$-adic setting (see [35, example 4.26]). In [37], it was shown that the set of functions $\mathbb{Q}_{p} \rightarrow \mathbb{Q}_{p}$ with $f^{\prime} \equiv 0$ that are not constant (or locally constant) on any ball is $\mathfrak{c}$-lineable. We show that this can be improved by restricting ourselfs to the Lipschitzian functions. It should be noted that in real analysis, the Lipschitz functions of order $\alpha>1$ are trivial.

Theorem 4.3 For every $\mathbb{K} \in\left\{\mathbb{Z}_{p}, \mathbb{Q}_{p}\right\}$, the family of non-locally constant functions $f: \mathbb{K} \rightarrow$ $\mathbb{Q}_{p}$ whose derivative is the zero function and $f$ belongs to $\operatorname{Lip}_{\alpha}\left(\mathbb{K} \rightarrow \mathbb{Q}_{p}\right)$ for every $\alpha>0$ is $\mathrm{c}$-lineable.

Proof Fix $\mathbb{K} \in\left\{\mathbb{Z}_{p}, \mathbb{Q}_{p}\right\}$. Given $q \in \mathbb{P}$, let us define the function $f_{q}: \mathbb{K} \rightarrow \mathbb{Z}_{p}$ as: for every $x=\sum_{n=r}^{\infty} a_{n} p^{n}$, with $r \in \mathbb{Z}$, we have $f_{q}(x)=\sum_{n=0}^{\infty} a_{n} p^{q^{(n+1) !}}$. For every $P \in \mathcal{P}$, define $F_{P}: \mathbb{K} \rightarrow \mathbb{Z}_{p}$ by $F_{P}=\sum_{q \in P} f_{q}$. Once again we have that $F_{P}$ is well defined for every $P \in \mathcal{P}$ since, by fixing $P \in \mathcal{P}$, the sequence $\left(f_{q}(x)\right)_{q \in P}$ converges for every $x \in \mathbb{Z}_{p}$. Indeed, we have $\left|f_{q}(x)\right|_{p} \leq p^{-q} \rightarrow 0$ as $q \rightarrow \infty$ for any $x \in \mathbb{K}$.

We will prove that the set $\left\{F_{P}: P \in \mathcal{P}\right\}$ is a family of linearly independent functions over $\mathbb{Q}_{p}$ such that any non-zero linear combination of these functions over $\mathbb{Q}_{p}$ is non-locally constant, belongs to $\operatorname{Lip}_{\alpha}\left(\mathbb{Z}_{p} \rightarrow \mathbb{Q}_{p}\right)$ for every $\alpha>0$ and its derivative is the zero function. 
It is clear that the functions $F_{P}$ and any non-zero linear combination of these functions is non-locally constant. Now we will show that they belong to $\operatorname{Lip}_{\alpha}\left(\mathbb{K} \rightarrow \mathbb{Q}_{p}\right)$ for any $\alpha>0$. Fix $\alpha>0$. For any distinct $x, y \in \mathbb{K}$ such that $|x-y|_{p}=p^{t}$ for some $t \in \mathbb{Z}$ we have that $\frac{\left|F_{P}(x)-F_{P}(y)\right|_{p}}{|x-y|_{p}^{\alpha}}=p^{-\tilde{q}^{(t+1) !}+\alpha t} \rightarrow 0$ as $t \rightarrow \infty$, and where $\tilde{q}=\min \{q \in P\}$. Hence, notice that the latter proves that $F_{P} \in \operatorname{Lip}_{\alpha}\left(\mathbb{K} \rightarrow \mathbb{Q}_{p}\right)$, and in the case $\alpha=1$ we have that $F_{P}^{\prime} \equiv 0$.

Now let $F=\sum_{j=1}^{n} b_{j} F_{P_{j}}$, where $n \in \mathbb{N}, b_{j} \in \mathbb{Q}_{p} \backslash\{0\}$ for any $1 \leq j \leq n$ and $P_{1}, \ldots, P_{n}$ are $n$ distinct elements of $\mathcal{P}$. Assume that $F$ is the zero function, then by fixing $q \in P_{1} \cap P_{2}^{c} \cap \cdots \cap P_{n}^{c}$ we have that $F\left(p^{q}\right)=b_{1} p^{q^{(q+1) !}}=0$ if and only if $b_{1}=0$, a contradiction. Now, it is easy to see that $F \in \operatorname{Lip}_{\alpha}\left(\mathbb{K} \rightarrow \mathbb{Q}_{p}\right)$ for any $\alpha>0$ and $F^{\prime} \equiv 0$.

In [41, p. 200], by using Mahler series, Mahler constructs a continuous function that is not differentiable only at a point but has continuous derivative elsewhere. By a totally different example, we show that the set of such functions is $\mathfrak{c}$-lineable.

Theorem 4.4 The family of continuous functions $\mathbb{Z}_{p} \rightarrow \mathbb{Q}_{p}$ that are not differentiable only at a point but have continuous derivative elsewhere is $\mathfrak{c}$-lineable.

Proof For any $q \in \mathbb{P}$, let us define $f_{q}: \mathbb{Z}_{p} \rightarrow \mathbb{Z}_{p}$ in the following way:

$$
f_{q}(x)= \begin{cases}p^{q^{n}} & \text { if } x \in S\left(0, p^{-q^{n+1}}\right) \text { for some } n \in \mathbb{N} \\ 0 & \text { otherwise. }\end{cases}
$$

The function $f_{q}$ is locally constant at every point except 0 , i.e., for every $x \in \mathbb{Z}_{p}$, there exists a neighborhood $V_{x}$ of $x$ such that $f_{q} \uparrow V_{x}$ is constant. Indeed, let $x \in \mathbb{Z}_{p}$, then we have two cases:

Case (1): If $x \in S\left(0, p^{-q^{n+1}}\right)$ for some $n \in \mathbb{N}$, then $f_{q} \uparrow S\left(0, p^{-q^{n+1}}\right) \equiv p^{q^{n}}$. Since $S\left(0, p^{-q^{n+1}}\right)$ is open for every $\varepsilon<p^{-q^{n+1}}$ we have that $B(x, \varepsilon) \subset$ $S\left(0, p^{-q^{n+1}}\right)$. Thus, $f_{q} \uparrow B(x, \varepsilon) \equiv p^{q^{n}}$.

Case (2): If $x \notin S\left(0, p^{-q^{n+1}}\right)$ for every $n \in \mathbb{N}$, then $x \in S\left(0, p^{-k}\right)$ for some $k \in$ $\mathbb{N}_{0} \backslash\left\{p^{-q^{n+1}}: n \in \mathbb{N}\right\}$. Applying the same arguments as in Case (1) we have that $f_{q}$ is identically zero on some neighborhood of $x$.

Moreover, $f_{q}$ is not locally constant at 0 since for every $\varepsilon \in\left\{p^{-k}: k \in \mathbb{N}_{0}\right\}$, there exists distinct $n, m \in \mathbb{N}$ such that $p^{-q^{n+1}}<\varepsilon$ and $p^{-q^{m+1}}<\varepsilon$. Hence, the spheres $S\left(0, p^{-q^{n+1}}\right)$ and $S\left(0, p^{-q^{m+1}}\right)$ are contained in $B(0, \varepsilon)$, which shows that $f_{q}$ takes at least two distinct values in $B(0, \varepsilon)$. (In fact, it takes infinitely many values.) Therefore, we have proven that $f_{q}$ is differentiable at every point except maybe 0 (we will see later that it is not differentiable at 0 ). However, $f_{q}$ is continuous at 0 (obviously it is continuous at every other point $x \neq 0$ since $f_{q}$ is differentiable at $x \neq 0$ ). Indeed, let $\varepsilon \in\left\{p^{-k}: k \in \mathbb{N}_{0}\right\}$ and take $n \in \mathbb{N}_{0}$ such that $p^{-q^{n}}<\varepsilon$. For any $x \in \mathbb{Z}_{p}$ with $|x|_{p}<p^{-q^{n+1}}$ we have $\left|f_{q}(x)\right|_{p}<p^{-q^{n}}<\varepsilon$. Now, $f_{q}$ is not differentiable at 0 since

$$
\frac{\left|f_{q}\left(p^{q^{n+1}}\right)\right|_{p}}{\left|p^{q^{n+1}}\right|_{p}}=p^{q^{n+1}-q^{n}}=p^{q^{n}(q-1)} \rightarrow \infty,
$$

as $n \rightarrow \infty$. 
For every $P \in \mathcal{P}$, define the function $F_{P}=\sum_{q \in P} f_{q}$. Using similar arguments used before, we see that $F_{P}$ is well defined. Moreover, by applying similar arguments used to prove that $f_{q}$ is continuous everywhere and differentiable at every point $x \neq 0$, we have that $F_{P}$ is differentiable at every point $x \neq 0$ and hence continuous at every point $x \neq 0$. But also continuous at 0 since $F_{P}$ is the uniform limit of the sequence of continuous functions $\left(\sum_{\substack{q \in P \\ q \leq k}} f_{q}\right)_{k \geq 2}$. Furthermore, the functions $F_{P}$ are not differentiable at 0 . To see this, fix $q \in P$. Then,

$$
\frac{\left|F_{P}\left(p^{q^{n+1}}\right)\right|_{p}}{\left|p^{q^{n+1}}\right|_{p}}=p^{q^{n}(q-1)} \rightarrow \infty
$$

as $n \rightarrow \infty$.

Also the functions $F_{P}$ are linearly independent over $\mathbb{Q}_{p}$ (apply similar arguments used in other proofs of this work). Finally, any non-zero linear combination over $\mathbb{Q}_{p}$ of the functions $F_{p}$ satisfies the desires properties. Indeed, take $r \in \mathbb{N}$ distinct elements of $\mathcal{P}$. Namely, $P_{1}, \ldots, P_{r}$, and take $F=\sum_{j=1}^{n} a_{j} F_{P_{j}}$, where $a_{j} \in \mathbb{Q}_{p} \backslash\{0\}$. Clearly $F$ is continuous everywhere and differentiable at every point $x \neq 0$. Now, fix $q \in P_{1} \cap P_{2}^{c} \cap \cdots \cap P_{r}^{c}$. Notice that $F\left(p^{q^{n+1}}\right)=a_{1} p^{q^{n}} \neq 0$, then

$$
\frac{\left|F\left(p^{q^{n+1}}\right)\right|_{p}}{\left|p^{q^{n+1}}\right|_{p}}=\left|a_{1}\right| p^{q^{n}(q-1)} \rightarrow \infty,
$$

as $n \rightarrow \infty$, which shows that $F$ is not differentiable at 0 .

\section{5 -adic sequence spaces and failure of the Cesàro and Hahn-Banach theorems}

In this section we present some results about lineability, algebrability and spaceability of some subsets of the space of $p$-adic sequences and conclude with a result concerning the failure of the Hahn-Banach theorem in the $p$-adic setting. To begin, we give an improvement of [36, proposition 2.1].

Theorem 5.1 Let $\mathbb{K}$ be a non-Archimedean field with non-trivial valuation. If $\ell_{\infty}$ and $c_{0}$ are defined over $\mathbb{K}$, then the set $\ell^{\infty} \backslash c_{0}$ is $\mathfrak{c}$-spaceable.

Proof For every $N \in \mathcal{N}$, let us define the sequence $x_{N}$ as follows: for every $n \in \mathbb{N}$,

$$
x_{N}(n)= \begin{cases}1 & \text { if } n \in N \\ 0 & \text { otherwise }\end{cases}
$$

Notice that the sequences in the set $\left\{x_{N}: N \in \mathcal{N}\right\}$ are linearly independent over $\mathbb{K}$ since $\mathcal{N}$ is a family of independent subsets of $\mathbb{N}$. Take $V=\operatorname{span}\left\langle\left\{x_{N}: N \in \mathcal{N}\right\}\right\rangle$. Clearly, any $x \in V$ is bounded. Also, if $x$ is not the zero sequence, then $x$ does not converge to 0 . Indeed, assume that $x=\sum_{i=1}^{m} a_{i} x_{N_{i}}$, where $a_{i} \in \mathbb{K} \backslash\{0\}$ and $N_{i} \in \mathcal{N}$ for every $1 \leq i \leq m$. Then, for every $n \in N_{1} \cap N_{2}^{c} \cap \cdots \cap N_{j}^{c}$ we have $x(n)=a_{1}$, i.e., $x$ restricted to the infinite set $N_{1} \cap N_{2}^{c} \cap \cdots \cap N_{m}^{c}$ is a constant non-zero infinite sequence.

Now, let $x \in \bar{V} \backslash\{0\}$, then there exists $\left(s_{k}\right)_{k \geq 1} \subset V \backslash\{0\}$ converging (uniformly) to $x$, i.e., $\left\|s_{k}-x\right\|_{\infty} \rightarrow 0$ as $k \rightarrow \infty$. We will prove that $x \in \ell^{\infty} \backslash c_{0}$. Clearly, $x$ is bounded 
since $\left(s_{k}\right)_{k \geq 1} \subset \ell^{\infty}$. As $x$ is not the zero sequence, there exists $n_{0} \in \mathbb{N}$ such that $x\left(n_{0}\right) \neq 0$, that is, there exists $r \in(0, \infty)$ such that $\left|x\left(n_{0}\right)\right|_{p}=r$. Thus, there exists $k_{0} \in \mathbb{N}$ such that $\left\|s_{k}-x\right\|_{\infty}<\frac{r}{2}$ for every $k \geq k_{0}$. For every $k \geq k_{0}$, let $N_{1, k}, \ldots, N_{m, k}$ be the sets that form $s_{k}$. Assume that $n_{0} \in N_{1, k}^{\varepsilon_{1}} \cap \cdots \cap N_{m, k}^{\varepsilon_{m}}$, where $\varepsilon_{i} \in\{0,1\}$ for every $1 \leq i \leq m$. Then, since $N_{1, k}^{\varepsilon_{1}} \cap \cdots \cap N_{m, k}^{\varepsilon_{m}}$ is infinite and the sequence $s_{k}$ restricted to $N_{1, k}^{\varepsilon_{1}} \cap \cdots \cap N_{m, k}^{\varepsilon_{m}}$ is a constant sequence, we have $s_{k}(n)=s_{k}\left(n_{0}\right)$ for infinitely many $n$. Notice that

$$
r=\left|x\left(n_{0}\right)-s_{k}\left(n_{0}\right)+s_{k}\left(n_{0}\right)\right| \leq \max \left\{\left|x\left(n_{0}\right)-s_{k}\left(n_{0}\right)\right|,\left|s_{k}\left(n_{0}\right)\right|\right\} .
$$

Therefore, since $\left|x\left(n_{0}\right)-s_{k}\left(n_{0}\right)\right|<\frac{r}{2}$, we have $\max \left\{\left|x\left(n_{0}\right)-s_{k}\left(n_{0}\right)\right|,\left|s_{k}\left(n_{0}\right)\right|\right\}=\left|s_{k}\left(n_{0}\right)\right|$. Thus, $\left|s_{k}\left(n_{0}\right)\right| \geq r$. Hence, $\left|s_{k}(n)\right| \geq r$ for infinitely many $n$. The latter implies that for infinitely many $n$ we have

$$
\frac{r}{2}>\left\|s_{k}-x\right\|_{\infty} \geq\left|s_{k}(n)-x(n)\right| \geq\left|s_{k}(n)\right|-|x(n)| \geq r-|x(n)| .
$$

This proves that $|x(n)|>\frac{r}{2}>0$ for infinitely many $n$. Therefore, $x$ does not converge to 0 in $\mathbb{K}$ and the proof is complete.

We pause to analyze the functions $(1+x)^{\alpha}$ where $x \in p \mathbb{Z}_{p}$ and $\alpha \in \mathbb{Z}_{p}$. By definition, the function $x \mapsto(1+x)^{\alpha}$ is defined as $(1+x)^{\alpha}:=F(x, \alpha):=\sum_{i=0}^{\infty}\left(\begin{array}{c}\alpha \\ i\end{array}\right) x^{i}$. Notice that by construction $(1+x)^{\alpha}$ is a function from $p \mathbb{Z}_{p}$ to $\mathbb{Z}_{p}$. The function $(1+x)^{\alpha}$ satisfies the following properties which can be found in [44, pp. 138-142]:

(i) $(1+x)^{\alpha}$ is differentiable and the derivative is $\alpha(1+x)^{\alpha-1}$.

(ii) For $x$ fixed, the sequence $\sum_{i=0}^{n}\left(\begin{array}{c}\alpha \\ i\end{array}\right) x^{i}$ converges uniformly (see [43]). Hence, since $p \mathbb{Z}_{p}$ is a compact metric space, the function $F(x, \alpha)$ is continuous in the second variable.

(iii) As a consequence of (ii) and the fact that $\mathbb{N}$ is dense in $\mathbb{Z}_{p}$, we have $(1+x)^{\alpha}(1+x)^{\beta}=$ $(1+x)^{\alpha+\beta}$ and $\left((1+x)^{\alpha}\right)^{\beta}=\left((1+x)^{\beta}\right)^{\alpha}=(1+x)^{\alpha \beta}$.

Let us continue by proving the following lemma which will be very useful in the sequel.

Lemma 5.2 If $\alpha_{1}, \ldots, \alpha_{n} \in \mathbb{Z}_{p} \backslash\{0\}$ are distinct, with $n \in \mathbb{N}$, then there is no linear combination $\sum_{i=1}^{n} \gamma_{i}(1+x)^{\alpha_{i}}$, with $\gamma_{i} \in \mathbb{Q}_{p} \backslash\{0\}$ for every $1 \leq i \leq n$, that is constant.

Proof We will prove it by induction on $n$. For $n=1$ we have that $F(x, \alpha)$ is a non-constant analytic function, and hence $\gamma F(x, \alpha)$ is not constant for every $\gamma \in \mathbb{Q}_{p} \backslash\{0\}$. We claim that the lemma is true up to $n-1$. Now, let $\alpha_{1}, \ldots, \alpha_{n} \in \mathbb{Z}_{p} \backslash\{0\}$ and assume that

$$
\sum_{i=1}^{n} \gamma_{i}(1+x)^{\alpha_{i}}=\gamma,
$$

for some $\gamma_{i} \in \mathbb{Q}_{p} \backslash\{0\}$ for every $1 \leq i \leq n$, and $\gamma \in \mathbb{Q}_{p}$. We have two cases:

Case (1): If $\gamma=0$, then $\sum_{i=2}^{n} \gamma_{i}(1+x)^{\alpha_{i}-\alpha_{1}}=\gamma_{1}$ for every $x \in p \mathbb{Z}_{p}$, which contradicts the inductive hypothesis (this can be done since $-1 \notin p \mathbb{Z}_{p}$ ).

Case (2): If $\gamma \neq 0$, then by differentiating (5.1) we have $\sum_{i=1}^{n} \gamma_{i} \alpha_{i}(1+x)^{\alpha_{i}-1}=0$. On the one hand, if $\alpha_{i} \neq 1$ for every $1 \leq i \leq n$, then we proceed as in Case (1). On the other hand, if $\alpha_{i}=1$ for some $1 \leq i \leq n$, then we would reach a contradiction with the inductive hypothesis.

Having these non-Archimedean tools, we are ready to show an improvement and generalization of [18, proposition 2.1] to the $p$-adic setting; see also [36, proposition 2.4]. 
Theorem 5.3 The subset of $\ell^{\infty} \backslash c_{0}$ defined over $\mathbb{Q}_{p}$ whose elements only have finitely many zero coordinates is strongly $\mathrm{c}$-algebrable.

Proof Let $\left\{z_{n}\right\}_{n \geq 1}$ be an enumeration of $\mathbb{Z}$ and for any $\alpha \in \mathbb{Z}_{p} \backslash\{0\}$ consider the sequence $\left(\left(1+p z_{n}\right)^{\alpha}\right)_{n \geq 1}$. Take $\mathcal{H}$ a Hamel basis of $\mathbb{Q}_{p}$ over $\mathbb{Q}$ contained in $\mathbb{Z}_{p} \backslash\{0\}$. Notice that the sequences $\left\{\left(\left(1+p z_{n}\right)^{h}\right)_{n \geq 1}: h \in \mathcal{H}\right\}$ are algebraically independent. Indeed, first of all, note that for every $n \in \mathbb{N}$, the function $P\left(\left(1+p z_{n}\right)^{h_{1}}, \ldots,\left(1+p z_{n}\right)^{h_{m}}\right)$ (where $P$ is a polynomial in $m \in \mathbb{N}$ variables with coefficients in $\mathbb{Q}_{p} \backslash\{0\}$ and without free term, and $h_{i} \in \mathcal{H}$ for every $1 \leq i \leq m)$ can be written as

$$
\sum_{i=1}^{k} \gamma_{i}\left(1+p z_{n}\right)^{\alpha_{i}}
$$

where $\gamma_{i} \in \mathbb{Q}_{p} \backslash\{0\}$ and $\alpha_{i} \in \mathbb{Z}_{p} \backslash\{0\}$ with $1 \leq i \leq k$ and $k \in \mathbb{N}$. By means of contradiction, assume that $\sum_{i=1}^{k} \gamma_{i}\left(1+p z_{n}\right)^{\alpha_{i}}=0$. Then, as $\mathbb{Z}$ is dense in $\mathbb{Z}_{p}$, we have that $\sum_{i=1}^{n} \gamma_{i}(1+x)^{\alpha_{i}}=0$ for every $x \in p \mathbb{Z}_{p}$ contradicting Lemma 5.2.

Clearly, for every $\gamma_{i} \in \mathbb{Q}_{p} \backslash\{0\}$ and $\alpha_{i} \in \mathbb{Z}_{p} \backslash\{0\}$ with $1 \leq i \leq k$, the sequence $\left(\sum_{i=1}^{k} \gamma_{i}\left(1+p z_{n}\right)^{\alpha_{i}}\right)_{n \geq 1}$ is bounded in $\mathbb{Q}_{p}$ by construction (in fact, we have $\left|\sum_{i=1}^{k} \gamma_{i}\left(1+p z_{n}\right)^{\alpha_{i}}\right|_{p} \leq \max _{1 \leq i \leq k}\left|\gamma_{i}\right|_{p}$, for every $\left.n \in \mathbb{N}\right)$. Now fix $x \in p \mathbb{Z}_{p}$. As $\left\{z_{n}\right\}_{n \geq 1}$ is dense in the set $\mathbb{Z}_{p}$, there exists a sequence $\left(z_{\sigma(n)}\right)_{n \geq 1}$ of $\left\{z_{n}\right\}_{n \geq 1}$ converging to $x$. Thus, since $\sum_{i=1}^{k} \gamma_{i}(1+p x)^{\alpha_{i}}$ is analytic (in particular, continuous) we have that $\left(\sum_{i=1}^{k} \gamma_{i}\left(1+p z_{\sigma(n)}\right)^{\alpha_{i}}\right)_{n \geq 1}$ converges to $\sum_{i=1}^{k} \gamma_{i}(1+p x)^{\alpha_{i}}$ in $\mathbb{Q}_{p}$. Furthermore, assume that $\left(\sum_{i=1}^{k} \gamma_{i}\left(1+p z_{\sigma(n)}\right)^{\alpha_{i}}\right)_{n \geq 1}$ tends to 0 in $\mathbb{Q}_{p}$. Then $\sum_{i=1}^{k} \gamma_{i}(1+p x)^{\alpha_{i}}=0$ with $x$ being an arbitrary element of $p \mathbb{Z}_{p}$ and, therefore, contradicting Lemma 5.2.

It is easy to see that the set of all conditionally convergent series of real numbers is algebrable with respect to the pointwise multiplication while, in [36], it was shown that in the $p$-adic setting the family of all sequences whose series is convergent but not absolutely convergent is $\left(\aleph_{0}, 1\right)$-algebrable in $c_{0}$. Here we prove a stronger and more optimal version of this result.

Theorem 5.4 In the space $c_{0}$ over $\mathbb{Q}_{p}$, the family of all sequences whose series is convergent but not absolutely convergent is strongly $\mathfrak{c}$-algebrable with respect to the pointwise multiplication.

Proof Let $\left(r_{n}\right)_{n \geq 1}$ be the sequence of exponents in the sequence $\left(t_{n}\right)_{n \geq 1}$ given in the proof of [36, proposition 4.2] and $\left\{z_{n}\right\}_{n \geq 1}$ be an enumeration of $\mathbb{Z}$. For every $\alpha \in \mathbb{Z}_{p} \backslash\{0\}$, consider the sequence $\left(p^{r_{n}}\left(1+p z_{r_{n}}\right)^{\alpha}\right)_{n \geq 1}$. Now, take $\mathcal{H}$ a Hamel basis of $\mathbb{Q}_{p}$ over $\mathbb{Q}$ contained in $\mathbb{Z}_{p} \backslash\{0\}$. We will prove that the sequences in $\left\{\left(p^{r_{n}}\left(1+p z_{r_{n}}\right)^{h}\right)_{n \geq 1}: h \in \mathcal{H}\right\}$ are algebraically independent by showing that any linear combination $s$ of products of the sequences $\left(p^{r_{n}}\left(1+p z_{r_{n}}\right)^{\alpha}\right)_{n \geq 1}$ is not absolutely convergent.

For any $n \in \mathbb{N}$, notice that $s(n)$ is of the form

$$
s(n)=\sum_{i=1}^{a} p^{\gamma_{i} r_{n}} \sum_{j=1}^{b_{i}} \beta_{i, j}\left(1+p z_{r_{n}}\right)^{\alpha_{i, j}},
$$

where $a \in \mathbb{N}, b_{i} \in \mathbb{N}$ and $\gamma_{i} \in \mathbb{N}$ with $\gamma_{i}<\gamma_{i+1}, \alpha_{i, j} \in \mathbb{Z}_{p} \backslash\{0\}$ and $\beta_{i, j} \in \mathbb{Q}_{p}$ with $\beta_{i, j} \neq 0$ for some pair $(i, j)$. First of all, $s$ converges in $\mathbb{Q}_{p}$ since 


$$
\begin{aligned}
|s(n)|_{p} & \leq \max _{1 \leq i \leq a}\left\{p^{-\gamma_{i} r_{n}}\left|\sum_{j=1}^{b_{i}} \beta_{i, j}\left(1+p z_{r_{n}}\right)^{\alpha_{i, j}}\right|_{p}\right\} \\
& \leq p^{-\gamma_{1} r_{n}} \max _{1 \leq i \leq a}\left|\sum_{j=1}^{b_{i}} \beta_{i, j}\left(1+p z_{r_{n}}\right)^{\alpha_{i, j}}\right|_{p} \\
& \leq p^{-\gamma_{1} r_{n}} \max _{1 \leq i \leq a} \max _{1 \leq j \leq b_{i}}\left|\beta_{i, j}\left(1+p z_{r_{n}}\right)^{\alpha_{i, j}}\right|_{p} \\
& \leq p^{-\gamma_{1} r_{n}} \max _{1 \leq i \leq a} \max _{1 \leq j \leq b_{i}}\left|\beta_{i, j}\right|_{p},
\end{aligned}
$$

tends to 0 as $n \rightarrow \infty$. Thus, $\sum_{n=1}^{\infty} s(n)$ converges in $\mathbb{Q}_{p}$ (see, [44, proposition 2.3.1]). Now, on the one hand, for every $1 \leq i \leq a$, by Lemma 5.2, there is $x \in \mathbb{Z}_{p}$ such that $\left|\sum_{j=1}^{b_{i}} \beta_{i, j}(1+p x)^{\alpha_{i, j}}\right|_{p}=p^{k_{i}}$ for some $k_{i} \in \mathbb{Z}$. Choose a subsequence $\left(r_{\sigma(n)}\right)_{n \geq 1}$ such that if $r_{\sigma(n)}=r_{m}$ for some $m \in \mathbb{N}$, then $r_{m}$ appears $p^{r_{\sigma(n)}^{2}}$ times in $\left(r_{\sigma(n)}\right)_{n \geq 1}$, and $\left(z_{r_{\sigma(n)}}\right)_{n \geq 1}$ converges to $x$ in $\mathbb{Q}_{p}$. Then, there exists $n_{i} \in \mathbb{N}$ such that

$$
p^{k_{i}}=\left|\sum_{j=1}^{b_{i}} \beta_{i, j}(1+p x)^{\alpha_{i, j}}\right|_{p}=\left|\sum_{j=1}^{b_{i}} \beta_{i, j}\left(1+p z_{r_{\sigma(n)}}\right)^{\alpha_{i, j}}\right|_{p},
$$

for every $n \geq n_{i}$. Therefore, for every $n \geq \max _{1 \leq i \leq a} n_{i}$, we have

$$
\left|p^{\gamma_{i} r_{\sigma(n)}} \sum_{j=1}^{b_{i}} \beta_{i, j}\left(1+p z_{r_{\sigma(n)}}\right)^{\alpha_{i, j}}\right|_{p}=p^{-\gamma_{i} r_{\sigma(n)}+k_{i}},
$$

for any $1 \leq i \leq a$. On the other hand, there exists $n_{*} \in \mathbb{N}$ such that $\gamma_{1} r_{\sigma(n)}-k_{1}<\gamma_{i} r_{\sigma(n)}-k_{i}$ for every $2 \leq i \leq a$ and every $n \geq n_{*}$. Hence, for every $n \geq \max \left\{n_{*}, \max _{1 \leq i \leq a} n_{i}\right\}$, we have $|s(\sigma(n))|_{p}=p^{-\gamma_{1} r_{\sigma(n)}+k_{1}}$ (see [35, remark 1.16]). Since $r_{\sigma(n)}$ appears $p^{r_{\sigma(n)}^{2}}$, we have that the sum of $|s(\sigma(n))|_{p}$ with the entries having $r_{\sigma(n)}$ is equal to $p^{r_{\sigma(n)}^{2}-\gamma_{1} r_{\sigma(n)}+k_{1}}$. Notice that there exits $n_{* *} \geq n_{*}$ such that $p^{r_{\sigma(n)}^{2}-\gamma_{1} r_{\sigma(n)}+k_{1}} \geq 1$ for every $n \geq n_{* *}$. As there are infinitely many $n$ having $|s(\sigma(n))|_{p} \geq 1$, we have that $s$ is not absolutely convergent.

A sequence $\left(x_{n}\right)_{n \geq 1}$ is Cesàro summable if its sequence of averages,

$$
\sigma_{n}:=\left(x_{1}+\ldots+x_{n}\right) / n,
$$

converges. Recall that, in the classical case, Cesàro's theorem states that if a sequence converges, then it is Cesàro summable to the same limit. This theorem does not have any $p$-adic analogous. In [36], Khodabendehlou and the second and fourth authors show that, in the $p$ adic setting, the set of all convergent sequences for which Cesàro's theorem fails is c-lineable, we now present two results about strong algebrability of the two extreme cases of Cesàro summability.

Theorem 5.5 In the space of all p-adic sequences over $\mathbb{Q}_{p}$, the family of convergent sequences that are not Cesàro summable is strongly c-algebrable.

Proof Let $\beta: \mathcal{N} \rightarrow(0,1)$ be a bijection. For every $N \in \mathcal{N}$, define the sequence $z_{N}$ as follows: for every $m \in \mathbb{N}$, we have 


$$
z_{N}(m)= \begin{cases}\left.p^{\lfloor\ln \beta(N)}(\ln (n))\right\rfloor & \text { if } m=p^{n} \text { with } n \in N, \\ 0 & \text { otherwise. }\end{cases}
$$

The sequences $\left\{z_{N}: N \in \mathcal{N}\right\}$ are algebraically independent. Indeed, take $P$ a polynomial of degree $k \geq 1$ in $p \in \mathbb{N}$ variables with coefficients in $\mathbb{Q}_{p} \backslash\{0\}$ and without free term, and $N_{1}, \ldots, N_{p} \in \mathcal{N}$. Let us define the sequence $(z(m))_{m \geq 1}=\left(P\left(z_{N_{1}}(m), \ldots, z_{N_{p}}(m)\right)\right)_{m \geq 1}$. Assume that $z$ is the zero sequence. For every $n \in N_{1} \cap N_{2} \cap \cdots \cap N_{p}$, notice that $z\left(p^{n}\right)$ is a linear combination of products of $p^{\left\lfloor\ln ^{\beta\left(N_{i}\right)}(\ln (n))\right\rfloor}$ with $1 \leq i \leq p$ (where the coefficients and exponents are fixed independently of $n$ ). As $N_{1} \cap \cdots \cap N_{p}$ has infinitely many elements, we have that not all of such linear combinations of products of $p^{\left\lfloor\ln ^{\beta\left(N_{i}\right)}(\ln (n))\right\rfloor}$ are 0 , which is absurd. Furthermore, it is easy to see that $z$ converges to 0 in $\mathbb{Q}_{p}$.

It remains to prove that $z$ is not Cesàro summable. Assume otherwise, that is, $\left(\frac{1}{m} \sum_{i=1}^{m} z(i)\right)_{m \geq 1}$ converges in $\mathbb{Q}_{p}$. Then, any subsequence of $\left(\frac{1}{m} \sum_{i=1}^{m} z(i)\right)_{m \geq 1}$ also converges in $\mathbb{Q}_{p}$. Hence,

$$
\left(\frac{1}{p^{n}} \sum_{i=1}^{p^{n}} z(i)\right)_{n \in N_{1} \cap \cdots \cap N_{p}} \text { and }\left(\frac{1}{p^{n}+1} \sum_{i=1}^{p^{n} z(i)}\right)_{n \in N_{1} \cap \cdots \cap N_{p}}
$$

converge to the same limit, say $a$, in $\mathbb{Q}_{p}$. Thus, $\left(\left|\frac{1}{p^{n}} \sum_{i=1}^{p^{n}} z(i)\right|_{p}\right)_{n \in N_{1} \cap \cdots \cap N_{p}}$ and $\left(\left|\frac{1}{p^{n}+1} \sum_{i=1}^{p^{n}} z(i)\right|_{p}\right)_{n \in N_{1} \cap \cdots \cap N_{p}}$ converge to $|a|_{p}$. Furthermore, notice that if $n \in N_{1} \cap$ $\cdots \cap N_{p}$, then

$$
\left|\frac{1}{p^{n}} \sum_{i=1}^{p^{n}} z(i)\right|_{p}=p^{n}\left|\sum_{i=1}^{p^{n}} z(i)\right|_{p} \text { and }\left|\frac{1}{p^{n}+1} \sum_{i=1}^{p^{n}} z(i)\right|_{p}=\left|\sum_{i=1}^{p^{n}} z(i)\right|_{p}
$$

converge to the same limit $|a|_{p}=0$. Indeed, for every $\varepsilon>0$, there exists $n_{0} \in N_{1} \cap \cdots \cap N_{p}$ such that $\left.|| \frac{1}{p^{n}} \sum_{i=1}^{p^{n}} z(i)\right|_{p}-|a|_{p}|=| p^{n}\left|\sum_{i=1}^{p^{n}} z(i)\right|_{p}-|a|_{p} \mid<\varepsilon$ for every $n \geq n_{0}$ with $n \in N_{1} \cap \cdots \cap N_{p}$. Thus, $\left.|| \sum_{i=1}^{p^{n}} z(i)\right|_{p}-p^{-n}|a|_{p} \mid<p^{-n} \varepsilon<\varepsilon$ for every $n \geq n_{0}$ with $n \in N_{1} \cap \cdots \cap N_{p}$. Now take $n \in \mathbb{N}$ with $n+1 \in N_{1} \cap \cdots \cap N_{p}$, then notice that $\sum_{i=1}^{p^{n+1}} z(i)=\sum_{i=1}^{p^{n}} z(i)+z\left(p^{n+1}\right)$. There exist $n_{1} \in \mathbb{N}, s \in \mathbb{N}$ sufficiently large and $\beta \in(0,1)$ sufficiently small such that $\left|z\left(p^{n+1}\right)\right|_{p}>p^{-s \ln ^{\beta}(n)}$ for every $n+1 \geq n_{1}$ with $n+1 \in N_{1} \cap \cdots \cap N_{p}$. Hence,

$$
\left|\sum_{i=1}^{p^{n}} z(i)+z\left(p^{n+1}\right)\right|_{p}>p^{-s \ln ^{\beta}(n)},
$$

for every $n+1 \geq n_{1}$ with $n+1 \in N_{1} \cap \cdots \cap N_{p}$. Thus, $\left|\frac{1}{p^{n+1}} \sum_{i=1}^{p^{n+1}} z(i)\right|_{p}>p^{-s \ln ^{\beta}(n)+n+1}$ which diverges as $n+1 \in N_{1} \cap \cdots \cap N_{p}$ tends to $\infty$, and we have reached a contradiction.

Proposition 5.6 In the space of all p-adic sequences over $\mathbb{Q}_{p}$, the family of convergent sequences that are Cesàro summable is $\mathrm{c}$-lineable. 
Proof For every $\alpha \in \mathbb{Z}_{p} \backslash\{0\}$, define the sequence $s_{\alpha}=\left((-1)^{n} p^{t(n)}(1+p t(n))^{\alpha}\right)_{n \geq 1}$, where

$$
t(n)= \begin{cases}\frac{n}{2} & \text { if } n \text { is even } \\ \frac{n+1}{2} & \text { otherwise. }\end{cases}
$$

Assume that $s=\sum_{i=1}^{m} \gamma_{i} s_{\alpha_{i}}$, where $m \in \mathbb{N}$ and $\gamma_{i} \in \mathbb{Q}_{p} \backslash\{0\}$ for every $1 \leq i \leq m$, is the zero sequence.

We can assume that the coefficients $\gamma_{i}$ that form the linear combination of $s$ belong to $\mathbb{Z}_{p} \backslash\{0\}$. (This can be done simply by multiplying $s$ by $p^{\max \left\{\operatorname{ord}\left(\gamma_{i}\right): 1 \leq i \leq m\right\}}$.) Therefore, we have that $\sum_{i=1}^{r} \gamma_{i}(-1)^{n} p^{t(n)}(1+p t(n))^{\alpha_{i}}=0$ for every $n \in \mathbb{N}$. This is equivalent to $\sum_{i=1}^{r} \gamma_{i}(1+p t(n))^{\alpha_{i}}=0$ for every $n \in \mathbb{N}$. Fix $x \in \mathbb{Z}_{p}$ arbitrary. Since $t(n)$ is surjective in $\mathbb{N}$, and $\mathbb{N}$ is dense in $\mathbb{Z}_{p}$, there exists a sequence $(\sigma(n))_{n \geq 1} \subset \mathbb{N}$ such that $(t(\sigma(n)))_{n \geq 1}$ converges to $x$. Hence, we have that $\left(\sum_{i=1}^{n} \gamma_{i}(1+p t(\sigma(n)))^{\alpha_{i}}\right)_{n \geq 1}$ converges to $\sum_{i=1}^{n} \gamma_{i}(1+p x)^{\alpha_{i}}$. As $x$ is an arbitrary element of $\mathbb{Z}_{p}$, we have that $\sum_{i=1}^{n} \gamma_{i}(1+p x)^{\alpha_{i}}=$ 0 for every $x \in \mathbb{Z}_{p}$, contradicting Lemma 5.2.

On the one hand, the sequences $s_{\alpha}$ and any linear combination converge to 0 in $\mathbb{Q}_{p}$. On the other hand, for every $n \in \mathbb{N}$, we have

$$
\frac{1}{n} \sum_{i=1}^{n}(-1)^{i} p^{t(i)}(1+p t(i))^{\alpha}= \begin{cases}0 & \text { if } n \text { is even, } \\ -\frac{1}{n} p^{t(n)}(1+p t(n))^{\alpha} & \text { otherwise. }\end{cases}
$$

Since $|n|_{p} \geq p^{-\lfloor\ln n / \ln p\rfloor}$ we have

$$
\left|-\frac{1}{n} p^{t(n)}(1+p t(n))^{\alpha}\right|_{p}=\left|-\frac{1}{n}\right|_{p}\left|p^{t(n)}\right|_{p}\left|(1+p t(n))^{\alpha}\right|_{p} \leq p^{\lfloor\ln (n) / \ln (p)\rfloor-t(n)} .
$$

Hence, $\frac{1}{n} \sum_{i=1}^{n}(-1)^{n} p^{t(n)}$ converges to 0 as $n \rightarrow \infty$ in $\mathbb{Q}_{p}$. It is easy to see that any linear combination of the sequences $s_{\alpha}$ is also Cesàro summable.

We conclude with a result about the Hahn-Banach theorem. Let us recall that if $\mathbb{K}$ is a non-Archimedean field with non-trivial valuation that is not spherically complete, then the Hahn-Banach theorem is not true over $\mathbb{K}$ (see [42, corollary 4.1.13] or [46, theorem 4.15]). It follows that the Hahn-Banach theorem cannot be extended to $\mathbb{C}_{p}$ since it is not spherically complete. We will show that the set of functionals for which the Hahn-Banach theorem fails in the non-Archimedean setting is c-lineable. To do so, let us consider first the following construction.

Let $\mathbb{K}$ be a non-Archimedean field with non-trivial valuation. Consider now $c_{0}$ and $\ell^{\infty}$ defined over $\mathbb{K}$. For every $N \in \mathcal{N}$, let us define $T_{N}: c_{0} \rightarrow \mathbb{K}$ as follows: for every $x=\left(x_{n}\right)_{n \geq 1} \in c_{0}$, we have

$$
T_{N}(x)=\sum_{n \in N} x_{n}
$$

Notice that $T_{N}$ is well defined for every $N \in \mathcal{N}$. Indeed, it is known that $\left(x_{n}\right)_{n \geq 1}$ converges to 0 in $\mathbb{K}$ if, and only if, $\sum_{n=1}^{\infty} x_{n}$ converges in $\mathbb{K}$ ([44, proposition 23.1]). Now, if $\left(x_{n}\right)_{n \geq 1}$ converges to 0 in $\mathbb{K}$, then so does the subsequence $\left(x_{n}\right)_{n \in N}$. Hence, $\sum_{n \in N} x_{n}$ converges in $\mathbb{K}$.

It is easy to see that $T_{N}$ is linear over $\mathbb{K}$ for every $N \in \mathcal{N}$. Let us prove that $T_{N}$ is continuous for every $N \in \mathcal{N}$. Fix $N \in \mathcal{N}$. For every $x=\left(x_{n}\right)_{n \geq 1} \in c_{0}$, we have 


$$
\left|T_{N}(x)\right|=\left|\sum_{n \in N} x_{n}\right| \leq \sup _{n \in N}\left|x_{n}\right| \leq \sup _{n \in \mathbb{N}}\left|x_{n}\right|=\|x\|_{\infty}<\infty .
$$

In fact, it is clear that $\left\|T_{N}\right\|=1$ for any $N \in \mathcal{N}$. Hence, $T_{N} \in c_{0}^{\prime}$ for any $N \in \mathcal{N}$.

Furthermore, the functionals $T_{N}$ are linearly independent. To see this, take $T=$ $\sum_{i=1}^{k} a_{i} T_{N_{i}}$, where $a_{i} \in \mathbb{K} \backslash\{0\}$ and with $N_{i} \in \mathcal{N}$ distinct for every $1 \leq i \leq k$. Assume that $T$ is the zero functional and fix $n_{0} \in N_{1} \cap N_{2}^{c} \cap \cdots \cap N_{k}^{c}$. Now consider $x=\left(x_{n}\right)_{n \geq 1}$ defined by

$$
x_{n}= \begin{cases}1 & \text { if } n=n_{0}, \\ 0 & \text { otherwise. }\end{cases}
$$

Clearly, $x \in c_{0}$. Moreover, $0=T(x)=a_{1}$, and we have a contradiction. Let $\mathcal{T}=$ $\operatorname{span}\left\langle T_{N}: N \in \mathcal{N}\right\rangle$. If $T \in \mathcal{T}$, then $T$ clearly belongs to $c_{0}^{\prime}$.

The following result is a refinement of [42, corollary 4.1.13] (see also [46, theorem 4.15]).

Lemma 5.7 Let $\mathbb{K}$ be a non-Archimedean field with non-trivial valuation. For every $T \in$ $\operatorname{span}\left\langle T_{N}: N \in \mathcal{N}\right\rangle \backslash\{0\}$, the following properties on $\mathbb{K}$ are equivalent:

(i) $\mathbb{K}$ is spherically complete.

(ii) The functional $T \in c_{0}^{\prime}$ can be extended to a functional $\bar{T} \in\left(\ell^{\infty}\right)^{\prime}$.

(iii) $\left(\ell^{\infty} / c_{0}\right)^{\prime} \neq\{0\}$.

Proof Fix $T=\sum_{i=1}^{k} a_{i} T_{N_{i}}$, where $a_{i} \in \mathbb{K} \backslash\{0\}$, and with $N_{i} \in \mathcal{N}$ distinct, for every $1 \leq i \leq k$.

(i) $\Rightarrow$ (ii) and (iii) $\Rightarrow(i)$ follow from [42, corollary 4.1.3] and [42, proposition 4.1.12], respectively.

It remains to prove $($ ii $) \Rightarrow($ iii $)$. By $($ ii $)$, let $\bar{T} \in\left(\ell^{\infty}\right)^{\prime}$ be an extension of $T$.

Let us denote $\widetilde{N}=\mathbb{N} \backslash \bigcap_{j=1}^{k} N_{j}^{0}$. Take $\left(k_{n}\right)_{n \geq 1} \subset \mathbb{N}$ the strictly increasing sequence such that $\widetilde{N}=\left\{k_{n}: n \in \mathbb{N}\right\}$. Consider $\phi: \mathbb{N} \rightarrow \widetilde{N}$ the bijection defined as $\phi(n)=k_{n}$, for every $n \in \mathbb{N}$, and define the auxiliary operator $R: \ell^{\infty} \rightarrow \ell^{\infty}$ as follows: for every $x=\left(x_{n}\right)_{n \geq 1} \in \ell^{\infty}$,

$$
R(x)=y=\left(y_{n}\right)_{n \geq 1},
$$

where, for every $n \in \mathbb{N}$, the coordinate $y_{n}$ is defined in the following way.

If $n=k_{m}$ for some $m \in \mathbb{N}$ with $k_{m} \in \bigcap_{j=1}^{k} N_{j}^{\varepsilon_{j}} \backslash\left\{\min \bigcap_{j=1}^{k} N_{j}^{\varepsilon_{j}}\right\}$, where $\varepsilon_{1}, \ldots, \varepsilon_{k} \in$ $\{0,1\}$ with $\varepsilon_{l} \neq 0$ for some $1 \leq l \leq k$, then

$$
y_{n}=x_{\max }\left\{k_{s} \in \bigcap_{j=1}^{k} N_{j}^{\varepsilon_{j}}: k_{s}<k_{m}\right\} .
$$

Otherwise, $y_{n}=0$.

Notice that $R$ is a continuous linear operator on $\ell^{\infty}$ such that $\bar{T} \circ R-\bar{T}=0$ on $c_{0}$. Assume that $\left(\ell^{\infty} / c_{0}\right)^{\prime}=\{0\}$, then $\bar{T} \circ R=\bar{T}$ on $\ell^{\infty}$. For every $x=\left(x_{n}\right)_{n \geq 1} \in \ell^{\infty}$, let us define inductively the sequence $z=\left(z_{n}\right)_{n \geq 1}$ in the following way.

Take $z_{1}=x_{1}$. For $n \geq 2$, assume that we have already defined $z_{r}$ for every $1 \leq r \leq n-1$. If $n \neq k_{m}$ for every $m \in \mathbb{N}$, or $n=k_{m}$ for some $m \in \mathbb{N}$ with $k_{m}=\min \bigcap_{j=1}^{k} N_{j}^{\overline{\varepsilon_{j}}}$ and where $\varepsilon_{1}, \ldots, \varepsilon_{k} \in\{0,1\}$ with $\varepsilon_{l} \neq 0$ for some $1 \leq l \leq k$, then $z_{n}=x_{n}$. If $n=k_{m}$ for some $m \in \mathbb{N}$ with $k_{m} \in \bigcap_{j=1}^{k} N_{j}^{\varepsilon_{j}} \backslash\left\{\min \bigcap_{j=1}^{k} N_{j}^{\varepsilon_{j}}\right\}$, where $\varepsilon_{1}, \ldots, \varepsilon_{k} \in\{0,1\}$ with $\varepsilon_{l} \neq 0$ for some $1 \leq l \leq k$, then 


$$
z_{n}=x_{k_{m}}+z_{\max }\left\{k_{s} \in \bigcap_{j=1}^{k} N_{j}^{\varepsilon_{j}}: k_{s}<k_{m}\right\} .
$$

Since $\mathbb{K}$ is non-Archimedean, we have that $z \in \ell^{\infty}$. Moreover, by construction, $x=z-R(z)$. Thus, $\bar{T}(x)=\bar{T}(z-R(z))=0$ for every $x \in \ell^{\infty}$. We have reached a contradiction since the latter implies that $\bar{T} \equiv 0$ on $\ell^{\infty}$, but $\bar{T} \uparrow c_{0}=T \not \equiv 0$.

We have proven the following result.

Theorem 5.8 If $\mathbb{K}$ is a non-spherically complete non-Archimedean field with non-trivial valuation, and $c_{0}$ and $\ell^{\infty}$ are defined over $\mathbb{K}$, then the family of functionals on $c_{0}^{\prime}$ that cannot be extended to a functional on $\left(\ell^{\infty}\right)^{\prime}$ is $\mathfrak{c}$-lineable.

Funding Open Access funding provided thanks to the CRUE-CSIC agreement with Springer Nature.

Open Access This article is licensed under a Creative Commons Attribution 4.0 International License, which permits use, sharing, adaptation, distribution and reproduction in any medium or format, as long as you give appropriate credit to the original author(s) and the source, provide a link to the Creative Commons licence, and indicate if changes were made. The images or other third party material in this article are included in the article's Creative Commons licence, unless indicated otherwise in a credit line to the material. If material is not included in the article's Creative Commons licence and your intended use is not permitted by statutory regulation or exceeds the permitted use, you will need to obtain permission directly from the copyright holder. To view a copy of this licence, visit http://creativecommons.org/licenses/by/4.0/.

\section{References}

1. Aizpuru, A., Pérez-Eslava, C., Seoane-Sepúlveda, J.B.: Linear structure of sets of divergent sequences and series. Linear Algebra Appl. 418(2-3), 595-598 (2006). https://doi.org/10.1016/j.laa.2006.02.041

2. Aizpuru, A., Pérez-Eslava, C., García-Pacheco, F.J., Seoane-Sepúlveda, J.B.: Lineability and coneability of discontinuous functions on $\mathbb{R}$. Publ. Math. Debr. 72(1-2), 129-139 (2008)

3. Araújo, G., Bernal-González, L., Muñoz-Fernández, G.A., Prado-Bassas, J.A., Seoane-Sepúlveda, J.B.: Lineability in sequence and function spaces. Stud. Math. 237(2), 119-136 (2017). https://doi.org/10. 4064/sm8358-10-2016

4. Aron, R.M., Bernal González, L., Pellegrino, D.M., Seoane Sepúlveda, J.B.: Lineability: The Search for Linearity in Mathematics Monographs and Research Notes in Mathematics, p. xix + 308. CRC Press, Boca Raton (2016)

5. Aron, R.M., Pérez-García, D., Seoane-Sepúlveda, J.B.: Algebrability of the set of non-convergent Fourier series. Stud. Math. 175(1), 83-90 (2006). https://doi.org/10.4064/sm175-1-5

6. Aron, R.M., Gurariy, V.I., Seoane-Sepúlveda, J.B.: Lineability and spaceability of sets of functions on $\mathbb{R}$. Proc. Am. Math. Soc. 133(3), 795-803 (2005). https://doi.org/10.1090/S0002-9939-04-07533-1

7. Aron, Richard M., Seoane-Sepúlveda, Juan B.: Algebrability of the set of everywhere surjective functions on $\mathbb{C}$. Bull. Belg. Math. Soc. Simon Stevin 14(1), 25-31 (2007)

8. Bartoszewicz, A., Głąb, S.: Strong algebrability of sets of sequences and functions. Proc. Am. Math. Soc. 141(3), 827-835 (2013). https://doi.org/10.1090/S0002-9939-2012-11377-2

9. Bartoszewicz, A., Bienias, M., Głąb, S.: Independent Bernstein sets and algebraic constructions. J. Math. Anal. Appl. 393(1), 138-143 (2012). https://doi.org/10.1016/j.jmaa.2012.03.007

10. Bastin, F., Conejero, J.A., Esser, C., Seoane-Sepúlveda, J.B.: Algebrability and nowhere Gevrey differentiability. Isr. J. Math. 205(1), 127-143 (2015). https://doi.org/10.1007/s11856-014-1104-1

11. Bernal-González, L., Cabana-Méndez, H.J., Muñoz-Fernández, G.A., Seoane-Sepúlveda, J.B.: On the dimension of subspaces of continuous functions attaining their maximum finitely many times. Trans. Am. Math. Soc. 373(5), 3063-3083 (2020). https://doi.org/10.1090/tran/8054

12. Bernal-González, L., Conejero, J.A., Murillo-Arcila, M., Seoane-Sepúlveda, J.B.: Highly tempering infinite matrices. Rev. R. Acad. Cienc. Exactas Fís. Nat. Ser. A Mat. RACSAM 112(2), 341-345 (2018). https://doi.org/10.1007/s13398-017-0385-8

13. Bernal-González, L., Fernández-Sánchez, J., Martínez-Gómez, M.E., Seoane-Sepúlveda, J.B.: Banach spaces and Banach lattices of singular functions. Stud. Math. 260(2), 167-193 (2021). https://doi.org/10. 4064/sm200419-7-9 
14. Bernal-González, L., Fernández-Sánchez, J., Seoane-Sepúlveda, J.B., Trutschnig, W.: Highly tempering infinite matrices II: from divergence to convergence via Toeplitz-Silverman matrices. Rev. R. Acad. Cienc. Exactas Fís. Nat. Ser. A Mat. RACSAM 114(4), 202-10 (2020). https://doi.org/10.1007/s13398020-00934-z

15. Bernal-González, L., Muñoz-Fernández, G.A., Rodríguez-Vidanes, D.L., Seoane-Sepúlveda, J.B.: Algebraic genericity within the class of sup-measurable functions. J. Math. Anal. Appl. 483(1), 123-576 (2020). https://doi.org/10.1090/tran/8054

16. Bernal-González, L., Pellegrino, D., Seoane-Sepúlveda, J.B.: Linear subsets of nonlinear sets in topological vector spaces. Bull. Am. Math. Soc. (N.S.) 51(1), 71-130 (2014). https://doi.org/10.1090/S02730979-2013-01421-6

17. Bonilla, A., Muñoz-Fernández, G.A., Prado-Bassas, J.A., Seoane-Sepúlveda, J.B.: Hausdorff and Box dimensions of continuous functions and lineability. Linear Multilinear Algebra 69(4), 593-606 (2021). https://doi.org/10.1080/03081087.2019.1612832

18. Cariello, D., Seoane-Sepúlveda, J.B.: Basic sequences and spaceability in $\ell_{p}$ spaces. J. Funct. Anal. 266(6), 3797-3814 (2014). https://doi.org/10.1016/j.jfa.2013.12.011

19. Ciesielski, K.C., Gámez-Merino, J.L., Pellegrino, D., Seoane-Sepúlveda, J.B.: Lineability, spaceability, and additivity cardinals for Darboux-like functions. Linear Algebra Appl. 440, 307-317 (2014). https:// doi.org/10.1016/j.laa.2013.10.033

20. Ciesielski, K.C., ., Seoane-Sepúlveda, J.B.: Differentiability versus continuity: restriction and extension theorems and monstrous examples. Bull. Am. Math. Soc. (N.S.) 56(2), 211-260 (2019). https://doi.org/ 10.1090/bull/1635

21. Ciesielski, K.C., Seoane-Sepúlveda, J.B.: A century of Sierpiński-Zygmund functions. Rev. R. Acad. Cienc. Exactas Fís. Nat. Ser. A Mat. RACSAM 113(4), 3863-3901 (2019). https://doi.org/10.1007/ s13398-019-00726-0

22. Enflo, P.H., Gurariy, V.I., Seoane-Sepúlveda, J.B.: Some results and open questions on spaceability in function spaces. Trans. Am. Math. Soc. 366(2), 611-625 (2014). https://doi.org/10.1090/S0002-99472013-05747-9

23. Fernández-Sánchez, J., Martínez-Gómez, M.E., Seoane-Sepúlveda, J.B.: Algebraic genericity and special properties within sequence spaces and series. Rocky Mountain J. Math (accepted) (2019) (preprint)

24. Fernández-Sánchez, J., Rodríguez-Vidanes, D.L., Seoane-Sepúlveda, J.B., Trutschnig, W.: Lineability and integrability in the sense of Riemann, Lebesgue, Denjoy, and Khintchine. J. Math. Anal. Appl. 492(1), 124433-16 (2020). https://doi.org/10.1016/j.jmaa.2020.124433

25. Fernández-Sánchez, J., Rodríguez-Vidanes, D.L., Seoane-Sepúlveda, J.B., Trutschnig, W.: Lineability, differentiable functions and special derivatives. Banach J. Math. Anal. 15(1), 18-22 (2021). https://doi. org/10.1007/s43037-020-00103-9

26. Fichtenholz, G., Kantorovich, L.: Sur les opérations dans l'espace des functions bornées. Stud. Math. 5, 69-98 (1934)

27. Gámez-Merino, J.L., Seoane-Sepúlveda, J.B.: An undecidable case of lineability in $\mathbb{R}^{\mathbb{R}}$. J. Math. Anal. Appl. 401(2), 959-962 (2013). https://doi.org/10.1016/j.jmaa.2012.10.067

28. García, D., Grecu, B.C., Maestre, M., Seoane-Sepúlveda, J.B.: Infinite dimensional Banach spaces of functions with nonlinear properties. Math. Nachr. 283(5), 712-720 (2010). https://doi.org/10.1002/mana. 200610833

29. García-Pacheco, F.J., Palmberg, N., Seoane-Sepúlveda, J.B.: Lineability and algebrability of pathological phenomena in analysis. J. Math. Anal. Appl. 326(2), 929-939 (2007). https://doi.org/10.1016/j.jmaa. 2006.03.025

30. Gouvêa, F.Q.: p-Adic Numbers, An Introduction, Universitext, 2, p. vi + 298. Springer, Berlin (1997). https://doi.org/10.1007/978-3-642-59058-0

31. Gurariy, V.I., Quarta, L.: On lineability of sets of continuous functions. J. Math. Anal. Appl. 294(1), 62-72 (2004). https://doi.org/10.1016/j.jmaa.2004.01.036

32. Gurariĭ, V.I.: Subspaces and bases in spaces of continuous functions. Dokl. Akad. Nauk SSSR 167, 971-973 (1966). (Russian)

33. Gurariı̆, V.I.: Linear spaces composed of everywhere nondifferentiable functions. C. R. Acad. Bulgare Sci. 44(5), 13-16 (1991). (Russian)

34. Jech, T.: Set theory, Springer Monographs in Mathematics, The Third Millennium Edition, Revised and Expanded. Springer, Berlin, pp. xiv+769 (2003)

35. Katok, S.: p-Adic Analysis Compared With Real, Student Mathematical Library, 37, American Mathematical Society, Providence; Mathematics Advanced Study Semesters, University Park, p. xiv+152 (2007)

36. Khodabendehlou, J., Maghsoudi, S., Seoane-Sepúlveda, J.B.: Algebraic genericity and summability within the non-Archimedean setting. Rev. R. Acad. Cienc. Exactas Fís. Nat. Ser. A Mat. RACSAM 115, 21 (2021). https://doi.org/10.1007/s13398-020-00961-w 
37. Khodabendehlou, J., Maghsoudi, S., Seoane-Sepúlveda, J.B.: Lineability and algebrability within $p$-adic function spaces. Bull. Belg. Math. Soc. Simon Stevin 27(5), 711-729 (2020)

38. Khodabandehlou, J., Maghsoudi, S., Seoane-Sepúlveda, J.B.: Lineability, continuity, and antiderivatives in the non-Archimedean setting. Can. Math. Bull. 64(3), 638-650 (2021). https://doi.org/10.4153/ S0008439520000715

39. Levine, B., Milman, D.: On linear sets in space $C$ consisting of functions of bounded variation, Russian, with English summary. Commun. Inst. Sci. Math. Méc. Univ. Kharkoff [Zapiski Inst. Mat. Mech.] (4) 16, 102-105 (1940)

40. Mahler, K.: An interpolation series for continuous functions of a $p$-adic variable. J. Reine Angew. Math. 199, 23-34 (1958). https://doi.org/10.1515/crll.1958.199.23

41. Mahler, K.: p-Adic Numbers and their Functions, Cambridge Tracts in Mathematics, p. xi+320. Cambridge University Press, Cambridge (1981)

42. Perez-Garcia, C., Schikhof, W.H.: Locally Convex Spaces Over Non-Archimedean Valued Fields, Cambridge Studies in Advanced Mathematics, 119, p. xiv+472. Cambridge University Press, Cambridge (2010)

43. Robert, A.M.: A Course in $p$-Adic Analysis, Graduate Texts in Mathematics, 198, pp. xvi+437. Springer, New York (2000)

44. Schikhof, W.H.: Ultrametric Calculus, An Introduction to $p$-Adic Analysis, Cambridge Studies in Advanced Mathematics, 4, p. viii+306. Cambridge University Press, Cambridge (1984)

45. Seoane-Sepúlveda, J.B.: Chaos and lineability of pathological phenomena in analysis. Thesis (Ph.D.), Kent State University, ProQuest LLC, Ann Arbor, MI, p. 139 (2006)

46. van Rooij, A.C.M.: Non-Archimedean Functional Analysis, Monographs and Textbooks in Pure and Applied Mathematics, 51, pp. x+404. Marcel Dekker, Inc., New York (1978)

Publisher's Note Springer Nature remains neutral with regard to jurisdictional claims in published maps and institutional affiliations.

\section{Authors and Affiliations}

\section{J. Fernández-Sánchez ${ }^{1}$. S. Maghsoudi ${ }^{2}$ D. L. Rodríguez-Vidanes ${ }^{3}$ (D) \\ J. B. Seoane-Sepúlveda ${ }^{4}$}

$凶 \quad$ J. B. Seoane-Sepúlveda jseoane@ucm.es

\section{J. Fernández-Sánchez}

juanfernandez@ual.es ; jfsjufesa@gmail.com

S. Maghsoudi

s_maghsodi@znu.ac.ir

D. L. Rodríguez-Vidanes

dl.rodriguez.vidanes@ucm.es

1 Grupo de investigación "Teoría de cópulas y aplicaciones", Universidad de Almería, Carretera de Sacramento s/n, 04120 Almería, Spain

2 Department of Mathematics, University of Zanjan, Zanjan 45371-38791, Iran

3 Departamento de Análisis y Matemática Aplicada, Facultad de Ciencias Matemáticas, Instituto de Matemática Interdisciplinar (IMI),, Universidad Complutense de Madrid, Plaza de Ciencias 3, 28040 Madrid, Spain

4 Departamento de Análisis Matemático y Matemática Aplicada, Facultad de Ciencias Matemáticas, Instituto de Matemática Interdisciplinar (IMI), Universidad Complutense de Madrid, Plaza de Ciencias 3, 28040 Madrid, Spain 